The Hamiltonian in the axial gauge may be written

$$
\begin{aligned}
\mathrm{H} & =\frac{1}{2} \int_{0} \mathrm{~d}^{3} x\left[\pi_{a}^{1} \pi_{a}^{1}+\pi_{a}^{2} \pi_{a}^{2}+\mathrm{A}_{a}^{0,3} \mathrm{~A}_{a}^{0,3}\right. \\
& \left.+\mathrm{A}_{a}^{1,3} \mathrm{~A}_{a}^{1,3}+\mathrm{A}_{a}^{2,3} \mathrm{~A}_{a}^{2,3}+!_{2}^{1,3} \mathrm{~F}_{a}^{1,2} \mathrm{~F}_{a}^{1,2}\right]
\end{aligned}
$$

and we may confirm that it generates the correct equations of motion of the independent variables in the Dirac brackets:

$$
\begin{aligned}
& \left\{\mathrm{A}_{a}^{i}(x), \mathrm{H}\right\}^{*}=\dot{\mathrm{A}}_{a}^{i}(x) \\
& \left\{\pi_{a}^{1}(x), \mathrm{H}\right\}^{*}=\Sigma_{3} \Sigma_{3} \mathrm{~A}_{a}^{1}-\Sigma_{2} \mathrm{~F}_{a}^{1 \cdot 2}+g \varepsilon_{a b c}\left(\pi_{b}^{1} \mathrm{~A}_{c}^{0}-\mathrm{F}_{b}^{1 \cdot 2} \mathrm{~A}_{c}^{*}\right) \\
& \left\{\pi_{a}^{2}(x), \mathrm{H}\right\}^{*}=\hat{\imath}_{3} \imath_{3} \mathrm{~A}_{a}^{2}+\hat{i}_{1} \mathrm{~F}_{a}^{12}+g \Sigma_{a b c}\left(\pi_{b}^{2} \mathrm{~A}_{c}^{0}+\mathrm{F}_{b}^{12} \mathrm{~A}_{c}^{1}\right) \text {. }
\end{aligned}
$$

We will forcgo the usual discussion of Poincaré covariance, etc.

We observe that the independent canonical variables in the axial gauge have canonical brackets equal to their Dirac brackets (6.72). There are no fields on the right hand side, and no ghost loops in the Feynman rules. On the other hand, the canonical variables in the radiation gauge have fields on the right hand side of their I irac brackets and ghost loops appear in the Feynman rules. It therefore appears that the canonical quantization procedure is best carried out in the axial gauge.

Finally, we remark that it is also instructive to analyze the lang-Mills field in the null-plane formalism. The gauge choice $A_{a}=0$ has properties similar to the radiation gauge and has ghost loops. The gauge $A_{a}=0$, on the other hand, gives simplifications parallel to those of the axial gauge (Tomboulis, 1973). In particular, the independent variables have field-independent Dirac brackets and no ghost loops result. We leave these analyses as exercises for the interested reader.

\title{
7. EINSTEIN'S THEORY OF GRAVITATION
}

As our last example of the application of Dirac's method to physical systems with constraints, we treat Einstein's theory of gravitation. Among the references giving elements of the Hamiltonian formulation of general relativity are Pirani and Schild (1950), Bergmann, Penfield, Schiller and Zatkis (1950), Pirani, Schild and Skinner (1952), Dirac (1958b, 1958c), Bergmann (1962), Arnowitt, Deser and Misner (1962), Hojman, Kuchař and Teitelboim (1973, I974), and Regge and Teitelboim (1974a, b). For a more complete account of the vast amount of literature, see Kuchar (1974). We observe that in Einstein's theory of gravitation, it is the expression of the theory in manifestly covariant form which causes constraints to exist among the canonical variables: the gauge-like freedom to make general coordinate transformations can then be exploited to reduce the number of independent degrees of freedom on a spacelike surface to two pairs of canonically conjugate variables per point. 
Our starting point will be the Hilbert action

$$
\mathrm{S}=\int\left(-{ }^{(4)} g\right)^{1 / 2(4)} \mathrm{R}(x) \mathrm{d}^{4} x
$$

for the pure gravitational field, where for convenience the units have been chosen in such a way that $G$, Newton's gravitational constant, takes on the value $(\mathrm{I} 6 \pi)^{-1}$. We deal with a hyperbolic Riemannian spacetime of signature $(-,+,+,+)$. Four-dimensional spacetime quantities carry an upper left index (4) whenever it is necessary to distinguish them from the threedimensional quantities to be introduced later. Greek indices run from o to 3 and Latin indices from I to 3 . Four-dimensional covariant differentiation is denoted by a semicolon, while its counterpart on a three-dimensional hypersurface is indicated by a slash. The Riemann tensor is defined as

$$
\mathrm{R}_{v \alpha \beta}^{\mu}=\Gamma_{v \beta, \alpha}^{\mu}-\Gamma_{v^{\alpha} \alpha, \beta}^{\mu}+\Gamma_{\sigma \alpha}^{\mu} \Gamma_{v^{\prime} \beta}^{\sigma}-\Gamma_{\sigma \beta}^{\mu} \Gamma_{v^{\prime} \alpha}^{\sigma}
$$

with

$$
\Gamma_{\alpha \beta}^{\mu .}=\frac{1}{2} g^{\mu \sigma}\left(g_{\sigma \alpha, \beta}+g_{\sigma \beta, \alpha}-g_{\alpha \beta, \sigma}\right) \text {. }
$$

The Ricci tensor and the scalar curvature are $\mathrm{R}_{\dot{\mu \nu},}=\mathrm{R}_{\mu \alpha \nu \nu}^{\alpha}$ and $\mathrm{R}=\mathrm{R}_{\mu,}^{\mu}$, respectively. The determinant of the metric is denoted by the letter $g$.

The appearance of the scalar curvature in (7.1) makes the action invariant under general coordinate transformations

$$
x^{\mu} \rightarrow x^{\prime \mu}(x)
$$

which change $g^{\mu \nu}(x)$ to

$$
g^{\prime \mu \nu}\left(x^{\prime}\right)=g^{\alpha \beta}(x) \frac{\partial x^{\prime \mu}}{\partial x^{\alpha}} \frac{\partial x^{\prime \nu}}{\partial x^{\beta}} .
$$

The theory is thus "already parametrized" in the sense of Section I. E. Note that the arbitrary coordinates denoted there by $u^{\prime \prime}$ are called $x^{\mu}$ here to conform with standard practice.

The action (7.I) describes the pure gravitational field. Addition of matter is straightfoward provided the matter action density contains no derivatives of $g_{\mu \nu}$, which is actually the case for particles and for the electromagnetic field.

\section{A. General Form of the Hamiltonian}

Einstein's equations are a second order partial differential system in the ten metric components $g_{\mu \nu}$ of a Riemannian spacetime. When looking at this system from a Hamiltonian point of view, one focuses his attention on a three-dimensional hypersurface embedded in the four-dimensional space. The state of the system is then given by specifying the value of certain fields defined on the surface; by means of the Hamiltonian, one is able to calculate the change in the field variables induced by a deformation of the hypersur- 
face. If $\mathrm{F}$ is an arbitrary functional of state we expect its changs under a deformation $\mathrm{N}^{\prime \prime}$ of the hypersurface to be of the form

$$
\mathrm{F}=\int \mathrm{d}^{3} x \mathrm{~N}^{+\prime \prime}(x) f_{\mu}(x)
$$

according to the general discussion on curved surfaces in Section (I. E.). Here $f_{u}(x)$ is some other functional of the canonical variables, the precise form of which will be examined in Section 7.C.

We can now use (7.2) to obtain information about the form of the Hamiltonian by relating the $\mathrm{N}^{\prime \prime}$ to the $g_{\alpha \beta}$. This is most easily done with the help

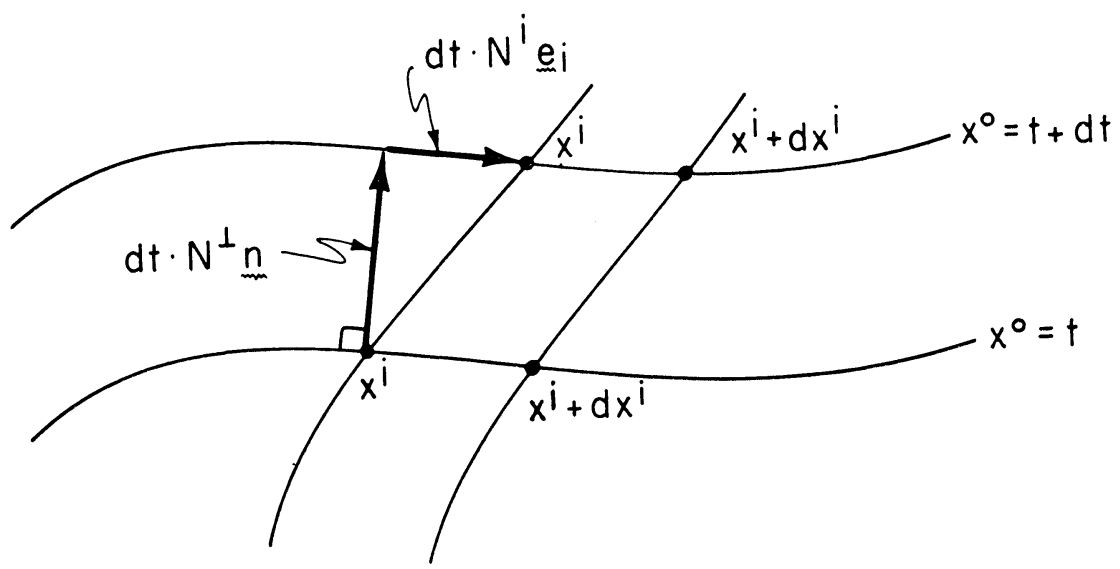

Fig. 7.I. The relation between the $N^{\mu}$ and the $g_{\alpha \beta}$ is obtained by evaluating the spacetime interval between the points with coordinates $\left(x^{i}, t\right)$ and $\left(x^{i}+\mathrm{d} x^{i}, t+\mathrm{d} t\right)$ in two different ways and comparing the resulting expressions for arbitrary d $x^{i}$, $\mathrm{d} t$. This leads to Eqs. $(7 \cdot 5)$.

of Fig. (7.I) which tells us that the spacetime distance between the points $\left(x^{i}, t\right)$ and $\left(x^{i}+\mathrm{d} x^{i}, t+\mathrm{d} t\right)$ can be expressed as

$$
\mathrm{d} s^{2}=-\left(\mathrm{N}^{1}\right)^{2} \mathrm{~d} t^{2}+g_{i j}\left(\mathrm{~N}^{i} \mathrm{~d} t+\mathrm{d} x^{i}\right)\left(\mathrm{N}^{i} \mathrm{~d} t+\mathrm{d} x^{i}\right)
$$

On the other hand, we know

$$
\mathrm{d} s^{2}=g_{\mu, \nu} \mathrm{d} x^{2 \mu} \mathrm{d} x^{\prime \prime}
$$

Upon comparison with Eq. (7.3), Eq. (7.4) shows that

$$
g_{i j} \mathrm{~N}^{i} \mathrm{~N}^{j}-\left(\mathrm{N}^{1}\right)^{2}=g_{00}
$$

and

$$
g_{i j} \mathrm{~N}^{j}=g_{0 i} .
$$

Conversely we can express $N^{\prime \prime}$ in terms of the $g_{\mu \nu}$ as

$$
\begin{gathered}
\mathrm{N}^{j}=g^{i j} g_{0 i}, \\
\mathrm{~N}^{1}=\left(-g^{00}\right)^{-\frac{1}{2}} .
\end{gathered}
$$


Note that $g^{i j}$ in $(7.6 a)$ is the inverse of the spatial metric $g_{i j}$ (i.e. $g^{k l} g_{\mid m}=\delta_{m}^{k}$ and is in general different from ${ }^{(4)} g^{i j}$. In fact the following useful relations hold (Arnowitt, Deser and Misner, 1962):

$$
\begin{aligned}
& \text { (4) } g^{i j}=g^{i j}-\mathrm{N}^{i} \mathrm{~N}^{j} /\left(\mathrm{N}^{\perp}\right)^{2} \\
& { }^{(4)} g^{(1 i}=N^{i} /\left(N^{1}\right)^{2} \\
& { }^{(4)} g_{00}=-\left[\left(\mathrm{N}^{1}\right)^{2}-g_{i,} \mathrm{~N}^{i} \mathrm{~N}^{i}\right] \\
& \left(-{ }^{(4)} g\right)^{\frac{1}{2}}=\mathrm{N}^{1} g^{\frac{1}{2}}
\end{aligned}
$$

Equations $(7 \cdot 5)$ tell us that $g_{00}$ and $g_{0 i}$ must enter in the Hamiltonian theory as arbitrary functions. This is so because the $N^{\prime \prime}$ must be prescribed from the outside in order to specifiy the deformation of the hypersurface; consequently one cannot expect Hamilton's equations to restrict them at all. Thus we learn that the non-trivial degrees of freedom are contained in the $g_{i j}$ and their conjugates $\pi^{i j}$.

It will save a little writing to work with the $\mathrm{N}^{\prime \prime}$ defined by (7.6) and the $g_{i j}$ as independent variables instead of using the $g_{0 \mu}$ and the $g_{i j}$. This change of variables is permissible because on account of $(7.5)$ and $(7.6)$, one can go back and forth between both sets of variables. If we denote by $\pi_{\mu}$ the conjugate to $N^{\prime \prime}$, then the fact that the $N^{+\prime \prime}$ are arbitrary tells us that $\pi_{\mu}$ must enter the Hamiltonian multiplied by an arbitrary function $\lambda^{\prime \prime}$ which will correspond to the time derivative of $N^{\prime \prime}$. This remark, together with $(7.2)$, indicates that we should expect the total Hamiltonian to be of the form

$$
\text { (7.10) } \mathrm{H}=\int \mathrm{d}^{3} x\left(\mathrm{~N}^{1} \mathscr{H}_{1}\left[g_{i j}, \pi^{i j}\right]+\mathrm{N}^{i} \mathscr{H}_{i}\left[g_{i j}, \pi^{i j}\right]+\lambda^{\mu} \pi_{i \mu}\right) \text {. }
$$

Since the $\pi_{j,}$ are multiplied by arbitrary functions, we must also expect the (first class) constraints

$$
\pi^{1 !} \approx 0
$$

to hold. Finally, since (7.I i ) must he preserved under surface deformations, we must have $\left\{\pi^{\prime \prime}, \mathrm{H}\right\} \approx 0$. From Eq. (7.IO), we find that $\left\{\pi^{\prime \prime}, \mathrm{H}\right\} \approx 0$ implies the additional constraints

$$
\mathscr{H}_{\mu} \approx 0 .
$$

The constraints (7.12) must be first class in order for the equations of motion with arbitrary $N^{+!}$to be consistent. Thus the constraints (7.II) would be primary constraints whereas (7.I2) would be secondary constraints.

Note that the above discussion applies generally for any relativistic Hamiltonian theory in a Riemannian space. It follows then that when matter is present the gravitational and matter parts of the Hamiltonian should be separately of the form $\int N^{\prime \prime} \mathscr{H}_{\mu}$ (supplemented of course by any extra first class constraints characteristic of the matter at hand). 
We see that we have obtained some insight into the structure of the Hamiltonian on general grounds. Equation (7.II) tells us that when working from the Lagrangian down to the Hamiltonian, we should try to add suitable divergences to the action density in (7.I) so that the resulting Lagrangian will contain no time derivatives of the $\mathrm{N}^{\mathrm{t}}$ (Anderson, 1958; De Witt, 1958; Dirac, 19586; Arnowitt, Deser and Misner, 1962). Furthermore the Lagrangian should have a very simple dependence on the $N^{\prime \prime}$ themselves in order to get a Hamiltonian of the form (7.IO). We thus need a way to analyze the spacetime curvature which clearly distinguishes the dependence of the curvature on the $g_{i, j}$ from the dependence on the $N^{* \prime}$. Such an analysis is best performed with the help of the embedding equations of Gauss and Codazzi which are reviewed in Appendix $B$.

\section{B. THE LAGRANGIAN}

Following Kuchar (197I), we now derive the desired Lagrangian beginning with the Hilbert action (7.I). Equation (B.5),

$$
\mathrm{K}_{u b b}=\left(2 N^{-1}\right)^{-1}\left(-g_{u b, 0}+\mathrm{N}_{a \mid b}+\mathrm{N}_{b, a}\right)
$$

relates the extrinsic curvature to the velocity $g_{a b, 0}$ of the dynamical coordinate $g_{a b}$ and to the functions $\mathrm{N}^{1}$ and $\mathrm{N}^{i}$ which describe the deformation of the hypersurface. Note that $g_{a b}$ itself also appears in this expression for $\mathrm{K}_{a b}$ through the Christoffel symbol hidden in the covariant derivatives $\mathrm{N}_{a^{\prime} b}+$ $+N_{b \mid a}$. There are, however, no time derivatives of $N^{1}$ and $N_{i}$ in (B.5.). Thus if we succeed in expressing the action density in terms of $g_{a b}$ and $\mathrm{K}_{a b}$, we will have achieved our goal of eliminating the velocities $\dot{N}^{1}$ and $\dot{N}_{i}$. Let us work in this direction with the help of the Gauss-Codazzi equations. In the basis $\left(\boldsymbol{n}, \boldsymbol{e}_{i}\right)$ used in (B.I), we have

$$
\begin{aligned}
{ }^{(1)} \mathrm{R}={ }^{(1)} \mathrm{R}^{\alpha_{\beta}^{\beta}{ }^{\beta}} & ={ }^{(1)} \mathrm{R}^{a b}{ }_{a b}{ }^{(1)} \mathrm{R}_{1 b}^{1 b}{ }^{(1)} \mathrm{R}_{a 1}^{a 1}= \\
& ={ }^{(1)} \mathrm{R}^{a b}{ }_{a b}-2{ }^{(1)} \mathrm{R}_{1 a \perp}^{a}= \\
& ={ }^{(1)} \mathrm{R}^{a b}{ }_{a b}-2{ }^{(1)} \mathrm{R}_{1 \times 1}^{\alpha}
\end{aligned}
$$

[In Eq. (7.I3), we have exploited the antisymmetry property $R_{\alpha \beta \gamma \delta}=-R_{\alpha \beta \delta \gamma}$ $=\mathrm{R}_{\xi \alpha \delta ;}$ of the Riemann tensor]. Now we use (B.I2) to transform (7.I3) into (7.I4)

$$
{ }^{(t)} \mathrm{R}=\mathrm{R}+\mathrm{K}^{2}-\mathrm{K}_{a b} \mathrm{~K}^{a b}-2{ }^{(t)} \mathrm{R}_{1 \times 1}^{\alpha}
$$

with $\mathrm{K} \approx \mathrm{K} u$.

The first three terms in (7.I4) are already of the form sought. We need to be concerned only about the remaining term ${ }^{(t)} \mathrm{R}_{1 \times 1}^{x}$. Our first step is to apply to the normal $n_{*}$ the commutation rule for the second covariant derivatives of an arbitrary vector, thus obtaining

$$
n_{\alpha ; \beta \gamma}-n_{\alpha ; \gamma \beta}={ }^{(4)} \mathrm{R}_{\alpha ; \beta \gamma}^{\delta} n_{\delta} .
$$


Next note that multiplication of (7.I5) by $n^{\mu}$ and subsequent contraction in the pairs $(\mu, \beta)$ and $(\alpha, \lambda)$ converts the right hand side of that equation into the desired quantity,

$$
n^{\beta}\left(n_{; \beta \gamma}^{\gamma}-n_{; \% \beta}^{\gamma}\right)={ }^{(4)} \mathrm{R}^{\delta \alpha} n_{\beta} n^{\beta}=\mathrm{R}_{\perp \perp \alpha}^{\alpha} .
$$

Now we rearrange the left hand side of (7.16) by means of the identities (see Appendix $B$ for a proof)

$$
\begin{gathered}
n^{\beta} n_{; \beta \gamma}^{\gamma}=\left(n^{\beta} n_{; \beta}^{\gamma}\right)_{; \gamma}-n_{; \gamma}^{\beta} n_{; \beta}^{\gamma}, \\
-n^{\beta} n_{; \gamma \beta}^{\gamma}=-\left(n^{\beta} n_{; \gamma ; \beta}^{\gamma}+n_{; \beta}^{\beta} n_{; \gamma}^{\gamma},\right. \\
n_{; \gamma}^{\beta} n_{; \beta}^{\gamma}=-\mathrm{K}_{a b} \mathrm{~K}^{a b}, \\
n_{; \beta}^{\beta}=-\mathrm{K} .
\end{gathered}
$$

Recalling that $\left(-{ }^{(1)} g\right)^{\frac{1}{2}}=\mathrm{N}^{-1} g^{\frac{1}{2}}$, we then olstain the relation

$$
\mathscr{L}_{\text {HILBERT }}=\left(-^{(1)} g\right)^{\frac{1}{2}(1)} \mathrm{R}=\mathscr{L}-\hat{i} \mathrm{~V}^{\alpha} / \hat{i} x^{\alpha},
$$

with

$$
\mathscr{L}=\mathrm{N}^{1} g^{\frac{1}{2}}\left(\mathrm{R}+\mathrm{K}_{a b} \mathrm{~K}^{a b}-\mathrm{K}^{2}\right)
$$

and

$$
\mathrm{V}^{\alpha}=2\left(-g^{(4)}\right)^{\frac{1}{2}}\left(n^{\beta} n_{; \beta}^{\alpha}-n^{\alpha} n_{; \beta}^{\beta}\right)
$$

Equation (7.20) shows that by adding to the Hilbert action density the divergence of $(7.2 \mathrm{I})$, we obtain a modified action density $\mathscr{L}$ which contains no time derivatives of $\mathrm{N}^{1}$ and $\mathrm{N}^{i}$ and which contains only first time derivatives of $g_{i j}$. Thus the Lagrangian

$$
\mathrm{L}=\int \mathrm{d}^{3} x \mathscr{L}=\int \mathrm{d}^{3} x \mathrm{~N}^{1} g^{\frac{1}{2}}\left(\mathrm{~K}_{a b} \mathrm{~K}^{a b}-\mathrm{K}^{2}+\mathrm{R}\right)
$$

will be our starting point for getting the Hamiltonian. However, before doing that, let us first take a closer look at the divergence in question. By means of Eqs. (7.5) - (7.9) and (B. 3) one can rewrite the quantity $\mathrm{V}_{, \alpha}^{\alpha}$ as (DeWitt, I967a)

$$
\mathrm{V}_{, \alpha}^{\alpha}=2\left(g^{\frac{1}{2}} \mathrm{~K}\right), 0_{0}-\left[g^{\frac{1}{2}}\left(\mathrm{KN}^{i}-g^{\frac{1}{2}} g^{i j} \mathrm{~N}^{1}, j\right)\right]_{,} .
$$

Equation (7.23) shows that both types of unwanted quantities, namely the first time derivatives of $\mathrm{N}^{\perp}, \mathrm{N}^{i}$ and the second time derivatives of $g_{i j}$, enter the Hilbert Lagrangian

$$
\mathrm{L}_{\mathrm{HILBERT}}=\int \mathrm{d}^{3} x\left(-{ }^{(4)} g\right)^{\frac{1}{2}(t)} \mathrm{R}
$$


in the form of a total derivative,

$$
\text { (7.25) }-\frac{\mathrm{d}}{\mathrm{d} t} 2 \int \mathrm{d}^{3} x g^{\frac{1}{2}} \mathrm{~K}
$$

and can therefore be eliminated altogether by omitting (7.25) from the Lagrangian.

The fact that one can eliminate the second time derivatives of $g_{i,}$ from the action is not surprising and was known long before the Hamiltonian formulation of general relativity was investigated. One can actually do even better and eliminate all second derivatives (temporal and spatial) of the ten $g_{\text {o. }}$ by adding a suitable divergence to $\mathscr{L}_{\text {HIIBBRT }}$ (Einstein's equations are of second order after all.). One arrives in this way at the so-called Einstein or "gamma-gamma" Lagrangian (see for example Landau and Lifshitz, I97I) which was used by Dirac (I9586) as a starting point for finding the gravitational Hamiltonian. What is much more remarkable and vital for the Hamiltonian formalism is the elimination of the first time derivatives of $\mathrm{N}^{\perp}$ and $\mathrm{N}^{i}$ [recall Section 7.A]. This possibility was explored by DeWitt (1958), Anderson (1958), and Dirac (19586).

\section{The Hamiltonian}

We proceed now to derive the exact form of the Hamiltonian for the gravitational field starting from the Lagrangian (7.22). Since no time derivatives of the $\mathrm{N}^{n}$ appear we immediately get the (first class) primary constraints

$$
\pi^{\mu}=\frac{\delta \mathrm{L}}{\delta \mathrm{N}^{\prime \mu}} \approx \mathrm{O}
$$

as we expected. The momenta $\pi^{i j}$ conjugate to $g_{i j}$ are by definition

$$
\pi^{i j}=\begin{gathered}
\delta \mathrm{L} . \\
\delta \dot{g}_{i j}
\end{gathered} .
$$

Recalling Eq. (B.5), we find after a simple calculation that

$$
\pi^{i j}=-g^{\frac{1}{2}}\left(\mathrm{~K}^{i j}-\mathrm{K} g^{i j}\right) \text {, }
$$

showing that the conjugate to the first fundamental form $g_{i j}$ is closely related to the second fundamental form $\mathrm{K}_{i j}$. Equation (7.28) can be inverted to express $\mathrm{K}^{i j}$ as a function of $g_{i j}$, which amounts to expressing the relocities $\dot{s}_{i j}$ as functions of the momenta and the dynamical coordinates. One thus gets from (7.28) that

$$
\mathrm{K}^{i j}=-g^{\frac{1}{2}}\left(\pi^{i j}-\frac{1}{2} \pi g^{i j}\right)
$$

where we have defined

$$
\pi=\pi_{i}^{i}=g_{i j} \pi^{i j}
$$

There are therefore no primary constraints other than (7.26). 
Next, using $(B .5),(7.22),(7.26)$ and $(7.29)$ we olstain for the Hamiltonian

$$
\mathrm{H}=\int \mathrm{d}^{3} x\left(\pi_{i, 1} \dot{\mathrm{N}}^{\prime \prime}+\pi^{i, j} \dot{g}_{i, j}\right)-\mathrm{L}=\int \mathrm{d}^{3} x\left(\mathrm{~N}^{\mathrm{L}} \mathscr{H}_{1}+\mathrm{N}^{i} \mathscr{H}_{i}\right)
$$

with

$$
\begin{gathered}
\mathscr{H}_{1}=\frac{1}{2} g^{-\frac{1}{2}}\left(g_{i k} g_{l l}+g_{i l} g_{j k}-g_{i j} g_{k l}\right) \pi^{i j} \pi^{k l}-g^{\frac{1}{2}} \mathrm{R}= \\
=g^{-\frac{1}{2}}\left(\pi_{i j} \pi^{i j}-\frac{1}{2} \pi^{2}\right)-g^{\frac{1}{2}} \mathrm{R}
\end{gathered}
$$

and

$$
\mathscr{H}_{i}=-2 g_{i k} \pi^{k j},{ }_{j}-\left(2 g_{k i, j}-g_{k j, i}\right) \pi^{k j}=-2 \pi_{i ! j}^{j} .
$$

In arriving at $(7.3 \mathrm{I})$, we have clropped the surface integral $2 \oint \mathrm{d}^{2} s_{j} \pi^{i, j} \mathrm{~N}_{i}$ from the right hand side of that equation.

To obtain the total Hamiltonian, we have to add to $(7.3 \mathrm{I})$ the first class primary constraints (7.26) multiplied by arlitrary functions. We therefore have

$$
\mathrm{H}=\int \mathrm{d}^{3} x\left(\mathrm{~N}^{1} \cdot \mathscr{H}_{\perp}+\mathrm{N}^{i} \cdot \mathscr{H}_{i}+\gamma_{\varkappa_{1}} \pi^{\mathrm{u}}\right)
$$

in agreement with the general discussion in Section (7.A).

Now to obtain the most general motion which is physically permissible, we ought to add to (7.33) the first class secondary constraints (7.12)

$$
\mathscr{H}_{\mu} \approx \mathrm{O}
$$

with arbitrary coefficients $u^{\text {: }}(x)$. However, since the $N^{\prime \prime}$ are arbitrary to start with, we do not get any additional freedom by doing this and we may as well set $u^{\prime \prime}=0$ without loss of generality ${ }^{(*)}$. The most general Hamiltonian is then just $(7 \cdot 33)$.

Equation (7.26) tells us that the degrees of freedom described by the variables $\left(\pi_{1, \mu}, N^{\prime \prime \prime}\right)$ are not physically important $\left(\pi^{i \cdot}\right.$ is constrained to be zero and $\mathrm{N}^{\prime \prime}$ is arbitrary). We can then drop these degrees of freedom from the phase space altogether and treat $\mathrm{N}^{\mu}$ henceforth as an arbitrary function $\mathrm{C}^{\prime \prime}(x)$ with vanishing brackets. Formally, such a procedure amounts to imposing the

$\left(^{*}\right)$ Geometrically speaking what gors on here is the following: Suppose some spacetime coordinate system $x^{\mu}$ is given. Then the $X: \mathscr{H}_{\mu}$ part of the Hamiltonian generates the changes in the canonical variables which correspond to passing from the surface $x^{0}=t$ to the surface $x^{0}=t+$ clt. Now the addition of the extra term $u^{\text {is }} \mathscr{H}_{2}$ would tell us that we are not forced to choose $x^{0}=t+\mathrm{d} t$ as our next surfice, but that we can go from $x^{0}=t$ to any infinitesimally close surface. However since the spacetime coordinate system $x^{\text {jo }}$ is arbitrary, we can make $x^{0}=t+\mathrm{d} t$ correspond to any given surface, and consequenty the freedom inherent in the $u^{i .}$ term can be considered to be already included in the arbitrariness of $x:$. A totally similar situation occurs with $A^{0}$ and the function $v_{2}$ in the Maxwell field (Section 5.A). The analogy is established by associating . $1^{0}$ with $N^{2}$, $v_{2}$ with $u^{2}$ and by making gauge tran formations $\left(A_{i} \rightarrow A_{i}+\partial_{i} \Lambda\right)$ take the place of surface deformations. 
second class constraint

$$
\mathrm{N}^{\prime \prime}-\mathrm{C}^{\prime \prime} \approx \mathrm{O}
$$

which makes the originally first class equation (7.26) become second class. The constraints (7.12) remain first class. One then passes to the Dirac l)racket by the usual procedure (which in this simple case amounts to working just with the $g_{i j}$ and $\pi^{i j}$ ) and takes (7.26) and (7.34) as strong equations.

At this stage the Poisson bracket is therefore

$$
\{\mathrm{F}, \mathrm{G}\}=\int \mathrm{d}^{3} x\left(\begin{array}{ccc}
\delta \mathrm{F} & \delta \mathrm{G} \\
\delta g_{i j} & \delta \pi^{i j}
\end{array}-\frac{\delta \mathrm{G}}{\delta g_{i j}} \frac{\delta \mathrm{F}}{\delta \pi^{i j}}\right) .
$$

When Eq. $(7 \cdot 35)$ is applied to the fundamental canonical variables themselves, we find

$$
\left\{g_{i j}(x), \pi^{k l}\left(x^{\prime}\right)\right\}=\delta_{i j}^{k l} \delta\left(x, x^{\prime}\right),
$$

as the only non-vanishing Poisson bracket. The symbol $\delta_{i j}{ }^{k l}$ in the right hand side of $(7.36)$ is a shorthand for

$$
\stackrel{1}{2}\left(\delta_{i}^{k} \delta_{j}^{l}+\delta_{i}^{l} \delta_{j}^{k}\right)
$$

and the Dirac $\delta$-function is defined as in (1.82) without recourse to the metric, by

$$
\int \mathrm{d}^{3} x \delta\left(x, x^{\prime}\right) f\left(x^{\prime}\right)=f(x)
$$

for an arbitrary scalar testing function.

If Eqs. (7.26) and $(7.34)$ are taken as strong equations, the Hamiltonian reads

$$
\mathrm{H}_{0}\left[g_{i j}, \pi^{k l}\right]=\int \mathrm{d}^{3} x\left(N^{1} \mathscr{H}_{1}+\mathrm{N}^{i} \mathscr{H}_{i}\right)
$$

and vanishes weakly due to the first class constraints (7.12).

The rate of change of an arbitrary functional $F$ of $g_{i j}$ and $\pi^{i j}$ is therefore given by

$$
\dot{\mathrm{F}}=\int \mathrm{d}^{3} x \mathrm{~N}^{\prime \prime}(x)\left\{\mathrm{F}, \mathscr{H}_{\mu}(x)\right\}
$$

so that Eq. (7.2) is explicitly verified.

\section{Asymptotically flat Space, Surface Integrals, Improved Hamiltonian, Poincaré Invariance At Spacelike Infinity}

We arrived in the last section at expression (7.38) for the gravitational Hamiltonian. We can now check whether Hamilton's equations correponding to $\mathrm{H}_{0}$ (together with the constraints (7.12)) reproduce Einstein's equations. It turns out that this is indeed the case provided one neglects certain surface terms. For a closed space one is certain that no complication could possibly 
arise from neglecting surface integrals, bacause every surface integral vanishes identically when the manifold has no boundary, So, for a closed space, $\mathrm{H}_{0}$ given by $(7.38)$ is the correct Hamiltonian as it stands. However for an open, asymptotically flat space certain subtleties arise and one comes to the conclusion (see below) that in such a case $\mathrm{H}_{0}$ must be supplemented by the addition of certain surface integrals at infinity in order to give the correct equations of motion (Regge and Teitelboim, $197+a, b$ ). The addition of the surface integrals also plays a crucial role when one imposes gauge conditions with the purpose of arriving at a canonical system having just two independent degrees of freedom per space point.

To see the role played by surface integrals, we start by observing that an essential requirement which must be met by an acceptable definition of the phase space of a dynamical system is that all physically reasonable solutions of the equations of motion must lie inside the phase space. If this is not true, the variational problem

$$
\delta \int\left(p_{i} \dot{q}^{i}-\mathrm{H}\right) \mathrm{d} t=\mathrm{o} .
$$

has no solutions because the extremal trajectories are not admitted among the original "competing curves" of the variational principle. Once the requirement of containing all extremal trajectories is met, one can enlarge the phase space at will but one cannot mutilate it arbitrarily.

In vacuum general relativity, after conditions (7.34) are imposed, a point in phase space is represented by twelve function variables $\left(g_{i j}, \pi^{k l}\right)$. Now any solution of Einstein's equations representing a physically reasonable, asymptotically flat spacetime behaves at spatial infinity in the Schwarzschild form

$$
\mathrm{d} s^{2} \underset{r \rightarrow \infty}{\longrightarrow}-\left(\mathrm{I}-\begin{array}{c}
\mathrm{M} \\
8 \pi r
\end{array}\right) \mathrm{d} \dot{t}^{2}+\left(\delta_{i j}+\begin{array}{cc}
\mathrm{M} & x^{i} x^{j} \\
8 \pi & r^{-3}
\end{array}\right) \mathrm{d} x^{i} \mathrm{~d} x^{j} .
$$

The phrase "physically reasonable" here means essentially that the total mass-energy of the system must be finite. This assumption will be satisfied if the system has been radiating (gravitationally or otherwise) during a finite time only. It is quite plausible, however, that one can adnit a more general situation in this context; one could allow a system which has been radiating during an infinite time, but doing so in such a way that the total amount of radiation remains finite. The precise form of the line element (7.39) can be altered by a change of coordinates, but no coordinate system exists such that, when $\mathrm{M}=0$, all components of the metric and its first spatial derivatives can be made to decrease at infinity faster than $r^{-1}$ and $r^{-2}$ respectively. It follows therefore that any definition of phase space has to contain metric functions such that

$(7.40 a)$

and

$(7.40 b)$

$$
g_{i j}-\delta_{i j} \sim r^{-1}
$$

$$
g_{i j, k} \sim r^{-2}
$$


We will need later a more precise definition of phase space, but the essential point is that one cannot do any better than (7.40).

Let us return now to Hamilton's principle, keeping the asymptotic behavior (7.40) in mind. When one deals with a continuous system like the present one, Hamilton's equations read

$(7 \cdot+\mathrm{I} a)$

$$
\dot{g}_{i j}(x)=\delta \text { (Hamiltonian) } / \delta \pi^{i j}(x)
$$

and

$$
\dot{\pi}^{i j}(x)=-\delta \text { (Hamiltonian) } / \delta g_{i j}(x),
$$

The functional derivatives appearing on the right hand side of (7.4I) are, by definition, the coefficients of $\delta g_{i j}$ and $\delta \pi^{i j}$ in a generic variation of the Hamiltonian, i.e., if

$\left(7 \cdot 4^{2}\right) \quad \delta$ (Hamiltonian) $=\int \mathrm{d}^{3} x\left\{\mathrm{~A}^{i j}(x) \delta g_{i j}(x)+\mathrm{B}_{i j}(x) \delta \pi^{i j}(x)\right\}$

then

$$
\delta(\text { Hamiltonian }) / \delta g_{i j}=\mathrm{A}^{i j}
$$

and

$$
\delta(\text { Hamiltonian }) / \delta \pi^{i j}=\mathrm{B}_{i j} .
$$

Thus, in order for Hamilton's equations to be defined at all, the variation of the Hamiltonian must be expressible in the form (7.42) for an arbitrary change in the phase space point $\left(g_{i j}, \pi^{k l}\right)$. We shall see now that $\delta \mathrm{H}_{0}$ (with $\mathrm{H}_{0}$ given by $(7.38)$ ) cannot be put in the form $(7.42)$ and that the Hamiltonian has to be amended by the addition of a surface integral in order for Hamilton's equations $(7 \cdot 4 \mathrm{I})$ to coincide with Einstein's equations. Introducing expressions $(7.32)$ into $(7.38)$ one gets for the change in $\mathrm{H}_{0}$, keeping all terms,

$$
\begin{aligned}
\delta \mathrm{H}_{0} & =\int \mathrm{d}^{3} x\left\{\mathrm{~A}^{i i}(x) \delta g_{i j}(x)+\mathrm{B}_{i j}(x) \delta \pi^{i j}(x)\right\}- \\
& -\oint \mathrm{d}^{2} s_{l} \mathrm{G}^{i j k l}\left(\mathrm{~N}^{1} \delta g_{i j ! k}-\mathrm{N}^{1}, k g_{i j}\right)- \\
& -\oint \mathrm{d}^{2} s_{l}\left\{2 \mathrm{~N}_{k} \delta \pi^{k l}+\left(2 \mathrm{~N}^{k} \pi^{i l}-\mathrm{N}^{l} \pi^{i k}\right) \delta g_{\jmath k}\right\}
\end{aligned}
$$

where

$$
\mathrm{G}^{i \jmath k l} \equiv \frac{1}{2} g^{\frac{1}{2}}\left(g^{i k} g^{j l}+g^{i l} g^{j k}-2 g^{i j} g^{k l}\right) .
$$

The coefficients $\mathrm{A}^{i j}$ and $\mathrm{B}_{i j}$ need not be explicitly written here [they may be found, for example, from the right hand sides of Eqs. $(7-3.15 a, b)$ of Arnowitt, Deser and Misner, 1962]. We need only to observe that in order for Hamilton's equations to reproduce Einstein's equations, one must identify $\mathrm{A}^{i j}$ and $\mathrm{B}_{i j}$ in Eq. (7.44) with the variational derivatives appearing in (7.43). 
This means that if the surface integrals in (7.44) would vanish then $H_{0}$ would the correct Hamiltonian. For a closed space this is indeed the case; however, for the open, asymptotically flat situation, some of the surface terms in (7.44) do not vanish due to the slow asympotic decrease characteristic of the gravitational field.

To deal with the surface integrals in $(7 \cdot 44)$ one needs a more complete specification of the asymptotic behavior than the one given by (7.40). An exhaustive discussion of the boundary conditions in question would take us away from our main line of development; we refer the reader to Regge and Teitelboim $(197+a, b)$ for a detailed treatment of this issue. We indicate explicitly here only that the lapse and shift functions $\mathrm{N}^{\prime \prime}$ are assumed to behave asymptotically as

$$
\mathrm{N}^{\prime \prime} \underset{r \rightarrow \infty}{ } \rightarrow \alpha^{\prime \prime}+\beta^{\prime \prime}, x^{\prime}
$$

with

$$
\beta_{r s}=-\beta_{s r} .
$$

[The Greek indices in $\alpha^{\prime \prime}$ and $\beta_{r}^{\mu}$ are raised and lowered with the Minkowskian metric $\left.r_{1 \times \beta}=\operatorname{diag}(-\mathrm{I}, \mathrm{I}, \mathrm{I}, \mathrm{I})\right]$.

Equation $(7.46)$ says that we allow asymptotic spacetime translations $\left(\alpha^{\prime \prime}\right)$, space rotations $\left(\beta_{s}^{r}\right)$ and boosts $\left(\beta^{\perp}\right.$ ) among the permissible deformations of the hypersurface. When Eq. $(7.46)$ is inserted into $(7.44)$ and the corresponding boundary conditions on $g_{i j}$ and $\pi^{i, j}$ are used, one finds

$$
\begin{aligned}
\delta \mathrm{H}_{0}= & \int \mathrm{d}^{3} x\left[\mathrm{~A}^{i j}(x) \delta g_{i, j}(x)+\mathrm{B}_{i, j}(x) \delta \pi^{i j}(x)\right] \\
& \alpha_{.}^{\prime \prime} \delta \mathrm{P}_{, \mu}-\frac{1}{2} \beta^{! \prime \prime} \delta \mathrm{I}_{i, \mu}
\end{aligned}
$$

with

$$
\mathrm{P}^{\perp}=\oint_{r \rightarrow \infty} \mathrm{d}^{2} s_{l}\left(g_{l, r, r}-g_{r, l l}\right)
$$$$
\mathrm{P}^{i}=-2 \oint_{r \rightarrow \infty} \mathrm{d}^{2} s_{l} \pi^{l i}
$$

$$
\mathrm{M}^{r s}=-2 \oint_{r \rightarrow \infty} \mathrm{d}^{2} s_{l}\left(x^{r} \pi^{l s}-x^{s} \pi^{l r}\right)
$$

$$
\mathrm{M}_{1,}=-2 \oint_{r \rightarrow \infty} \mathrm{d}^{2} s_{l}\left[x^{r}\left(g_{s l, s}-g_{s s, l}\right)-g_{r l}+g_{s s} \delta_{r l}\right] .
$$

Here we have defined.

$$
(7.50 a)
$$

$$
\beta_{1,}=-\beta_{, \perp} ; \quad M_{r_{1}}=\cdots \Lambda_{\perp^{r}},
$$

so that one has

$$
\beta_{\mu \nu}=-\beta_{\nu \mu} \quad ; \quad M_{\mu \nu}=-M_{\nu, \mu} .
$$


From (7.47) we see that $\mathrm{H}_{0}$ has well defined functional derivatives only when the surface integrals (which are in general different from zero) are absent from the right side of that equation, i.e., when $\alpha^{\mu}=\beta^{\mu \nu}=0$. Thus $H_{0}$ is a good Hamiltonian only for those deformations of the hypersurface which involve neither spacetime translations nor spacetime rotations at infinity. However, Eq. (7.47) itself suggests how to deal with the more general case: one simply passes the surface integrals in $(7.47)$ to the left hand side to obtain a new "improved" Hamiltonian

$$
\mathrm{H}=\mathrm{H}_{0}-\alpha^{\mu} \mathrm{P}_{\mu}+\frac{1}{2} \beta^{\mu \nu} \mathrm{M}_{\mu \nu} .
$$

The functional $\mathrm{H}$ defined by Eq. (7.5 I) has well defined functional derivatives and generates the correct equations of motion even when asymptotic Poincaré transformations are allowed among the permissible deformations of the hypersurface.

Now $\mathrm{H}_{0}$ vanishes weakly on account of (7.I2) implying that for any solution of the equations of motion, the numerical value of the Hamiltonian $(7.5 \mathrm{I})$ is

$$
\mathrm{H} \approx-\alpha^{\mu} \mathrm{P}_{\mu}+\frac{1}{2} \beta^{\mu \nu} \mathrm{M}_{\mu \nu}
$$

This expression is in general different from zero. When $\beta_{\mu \nu}=\alpha^{i}=0$ and $\alpha^{0}=\mathrm{I}$ one has $\mathrm{H}=\mathrm{P}^{\perp}$, which identifies $(7.48 a)$ as the energy. Similarly proceeding along the familiar lines of Noether's theorem one identifies expression $(7.486)$ as the linear momentum and (7.49) as the angular momentum. (See Appendix B.3). It is important to realize that the identification of $\mathrm{P}_{\mu}$ and $\mathrm{M}_{f \sigma}$ has been achieved without recourse to any special decomposition of the canonical variables or to a specific fixation of the spacetime coordinates, which makes obvious the "gauge independence" of these quantities (compare Arnowitt, Deser and Misner, I962).

Having written expression (7.48) and (7.49) for $\mathrm{P}^{\mu}$ and $\mathrm{M}_{\rho \sigma}$, one asks himself immediately whether these quatities have the proper behavior under asymptotic Poincaré transformations. The straightforward approach to answering this question would be to introduce the lapse and shift functions (7.46) into the equations of motion (7.41), but such a procedure would be a bit awkward. We prefer to follow instead a different route which sheds light on other aspects of the problem. Since the spacetime is asymptotically Minkowskian, we can introduce a system of rectangular spacetime coordinates at infinity. These coorrinates will be denoted by $y^{1}(\Lambda=0,1,2,3)$. The surface on which the state is defined will have, asymptotically, the equation $y^{\Lambda}=a^{\Lambda}+b^{\Lambda}{ }_{r} x^{r}$, with $b^{\Lambda}{ }_{r} b_{\Lambda s}=\delta_{r s}$ (this ensures that the coordinates $x^{r}$ become rectangular at infinity). Now, according to Dirac's procedure for dealing with curved surfaces described in Section (I.E), one should consider the "surface variables" describing the location of the surface on the same footing as the "truly dynamical" variables themselves. In the case of the gravitational field, the nonasymptotic parts of the "surface variables" are already 
implicitly included in the six canonical pairs $\left(g_{i j} ; \pi^{i j}\right)$, which exceed by four the required number for a theory with two "truly dynamical" degrees of freedom per point. As a consequence, one has the constraints $\mathscr{H}_{\mu} \approx$ o (Baierlein, Sharp and Wheeler, 1962; Kuchar, 1974). However, the asymptotic location of the surface, governed by ten independent quantities among the sixteen $a^{1}, b^{1}$, (remember $b^{-1}, b_{\lambda s}=\delta_{r s}$ ) cannot be determined from a knowledge of $g_{i j}$ and $\pi^{i j}$. One must therefore introduce $a^{\prime}$ and $b^{\prime}$, together with corresponding conjugate momenta $\pi_{\Lambda}, \pi_{\Lambda}{ }^{r}$ as additional canonical variables on the same footing as the $g_{i j}, \pi^{i j}$. After this is done, one will have a Hamiltonian formalism which is manifestly covariant under Poincaré transformations at infinity.

When we introduce ten new pairs of canonical varialles we must gain, unavoidably, ten new constraints. Each of these constraints will enter the Hamiltonian with an arbitrary Lagrange multiplier. The multipliers in question will describe the amount of hypersurface deformation at infinity anci will be given precisely by the $\alpha^{\prime \prime}$ and $\beta^{! \prime \prime}$ appearing in (7.39). The new, extended Hamiltonian, $\mathrm{H}_{\mathrm{E}}$, will then be of the form

$$
\begin{aligned}
& \mathrm{H}_{\mathrm{L}}=\int \mathrm{d}^{3} x \mathrm{~N}^{\mathrm{\mu}}(x) \mathscr{H}_{\mu}(x)+\alpha^{\prime \prime}\left(p_{\mu,}-\mathrm{P}_{\mu}\right)+\frac{1}{2} \beta^{\prime \prime \prime}\left(m_{\mu, \nu}+\mathrm{M}_{\mu, \nu}\right)= \\
& =\mathrm{H}+\alpha^{! \prime \prime} p_{! \alpha}+\stackrel{1}{2} \beta^{! \prime \prime \prime} m_{[\alpha \nu} \text {. }
\end{aligned}
$$

In addition to the $\mathscr{H}_{0 .} \approx 0$ equations, we find the new constraints

$$
(7.5+a) \quad t_{i, 2} \approx P_{\mu,},
$$

and

$$
m_{\mu \nu}+M_{\mu \nu} \approx 0
$$

The quantities $p_{i, 2}$ and $m_{1, \nu}$ are constructed from the asymptotic surface variables $a^{1}, b^{1}$, and their conjugates. The details of this construction are actually irrelerant - what matters are the Poisson brackets that $p_{\mu .}$ and $m_{j, y}$ satisfy. One may in fact show that the integrability conditions ( 1.83 ) of a Hamiltonian theory on curved surfaces imply that these quantities satisfy the algebra of the infinitesimal generators of the Poincaré group) (Regge and Teitelboim. $197+a, b)$. One thus has

$$
\begin{aligned}
& \left\{p_{\mu}, p_{\nu}\right\}=\mathrm{o}, \\
& (7.55 b) \quad\left\{p_{\mu \alpha}, m_{\sigma \sigma}\right\}=\eta_{i \mu \sigma} p_{p}-r_{i \mu \sigma} p_{\sigma \sigma}, \\
& (7.55 c) \quad\left\{m_{\mu \nu,}, m_{\sigma \sigma}\right\}=r_{i \mu \rho} m_{\nu \sigma}-r_{i, \rho} m_{\mu, \sigma}-r_{i \mu \sigma} m_{\nu, \rho}+r_{i v \sigma} m_{\mu, \rho} .
\end{aligned}
$$

where $r_{1 ! 2}$ is the Minkowski metric.

Once we know that $p_{\mu,}$ and $m_{\mu \nu}$ oley the Poincaré group brackets, it follows immediately from $(7.54)$ that $\mathrm{P}_{\mu,}$ and $\mathrm{M}_{\mu, 2,}$ transform in the correct way under an asymptotic Poincaré transformation; the only way in which the constraints $(7.54)$ could be preserved under surface deformations is for $t_{\text {:.. }}$ 
and $P_{p}\left(m_{y, y}\right.$ and - $\left.M_{i, y}\right)$ to crolve in the same fashion. The reader may wonder at this point why one does not just calculate the Poisson brackets of the $\mathrm{P}_{\mu \mu}$ and $\mathrm{M}_{z \sigma}$ among themselves instead of working with the extra variables $p_{\mu,}$ and $m_{\mu \nu}$. The answer is that these Poisson brackets do not cxist for the same reason that the functional derivatives of $\mathrm{H}_{0}$ given $b_{y}(7.38)$ do not exist. It is only the sum $(7.5 \mathrm{I})$ which has well defined Poisson brackets (*) [recall $(7 \cdot 47)]$. Geometrically speaking, the non-existence of functional derivatives of $P_{\mu}$ and $M_{r \sigma}$ follows from the impossibility of continuously deforming a surface at infinity without altering its shape elsewhere. (See Section (7.E) below for a discussion related to this issue).

We finish this section with the observation that the Hamiltonian $\mathrm{H}_{1}$ : given by $(7.53)$ is to open spaces what $\mathrm{H}_{0}$ given by $(7.5 \mathrm{I})$ is to closed spaces. In fact, both quantitics vanish weakly and their arguments contain, in each case, the full specification of the surface on which the state is defined. Correspondingly, it is the product of the space of the $g_{j j}, \pi^{i j}$ with the space spanned by the asymptotic surface variables $a^{1}, b^{1}$, and their conjugates which plays for open three-spaces the role that the $\left(g_{i j}, \pi^{i j}\right)$ space alone plays for compact three-surfaces.

\section{E. IFATTON OF THE SPACETIME COORDANATES GAl'iE,}

The equations of motion associated with the Hamiltonian $7.5 \mathrm{I}$ ) (lescribe the erolution of the system under arbitrary deformation of the hypersurface on which the field state is defined. Often one wants to answer a more limited question, such as how the system erolves along a one-parameter family of surfaces. Then one does not need the whole power of the theory based on the general Hamiltonian containing four arlitrary functions $\mathrm{N}^{-1}$ and $\mathrm{N}^{-i}$; a

*) To verify that the constraints $(7.54)$ are first class, one treats all of them at once, together with the $\mathscr{P}_{:} \approx 0$ equation, by writing

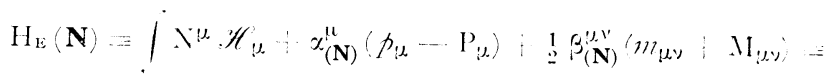

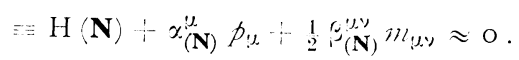

(The subscript $(\mathbf{N})$ in $\alpha^{\prime 2}$ and $\beta^{\prime \prime \prime}$ is intended to remind us that these quantities are related to Ne through the asymptotic formula $(7.46)$.) The statement that all constraints (i.e. $H_{0} \approx 0$ and $(7.54)$ are first class then reads

$$
\left\{\mathrm{H}_{\mathrm{L}}(\mathbf{S}), \mathrm{H}_{\mathrm{E}}(\boldsymbol{y})\right\}=\mathrm{H}_{\mathrm{E}}(\boldsymbol{\bullet})
$$

for any given $\$$ and $/ /$ and some $:$ Actually for the theory to be consistent with the Ri(mannian structure of sparctime (i.e. for the crolution to be path inclependent in the sense of Section (1.E.), = must be given by (Teitelboim. $1973 a, b$ )

$$
\begin{aligned}
& \stackrel{-1}{r}=-\left(\eta^{r} \xi_{, r}^{1}-\xi_{s}^{r} \eta_{, r}^{1}\right), \\
& \ddot{v}^{r}=-g^{r s}\left(r_{1}^{1} \xi_{, s}^{1}-\xi_{s}^{1} r_{1, s}^{1}\right)+\left(\xi^{s} r_{, s}^{r}-\eta_{1}^{r} \xi_{, s}^{r}\right)
\end{aligned}
$$

which is an alternative way of writing $\mathrm{Eq}(\mathrm{I} .83)$. This is the starting point for deriving $(7 \cdot 55)$. 
reduced Hamiltonian which is able to give only information one is asking for suffices. The reduced Hamiltonian theory is obtained by freezing the degrees of freedom corresponding to an arbitrary deformation of the surface. It has no constraints and a lesser number of degrees of freedom than the full theory. The gauge-like freedom to make arbitrary transformations of the spacetime coordinates is thereby destroyed.

It turns out that the procedure for fixing the coordinates is quite different depending on whether the three-space is open or closed; we will therefore treat each case separately.

\section{OPEN SPACES}

In this case the gauge freedom corresponds to the possibility of making arbitrary deformations of the surface while keeping its asymptotic shape unchanged.

We then want to impose additional constraints so that the previously first-class equations $\mathscr{H}_{\mu} \approx$ o which constrain the deformation generators to vanish become second class. After the additional constraints have been imposed the Hamiltonian will become just a surface integral

$$
I=-\alpha^{\mu} P_{\mu}+\frac{1}{2} \beta^{\mu \nu} M_{\mu \nu}
$$

according to (7.52). It will be possible to use $(7.56)$ as a meaningful Hamiltonian because, as we shall see below, the surface integral $(7.56)$ acquires well defined Poisson brackets (the Dirac brackets) after gauge conditions have been imposed, even though its functional derivatives with respect to $g_{i j}$ and $\pi^{i j}$ do not exist $\left.{ }^{*}\right)$.

Instead of immediately evaluating the Dirac brackets for the gauge conditions of interest from Eq. (I.58), we shall follow instead a somewhat indirect procedure. This will enable us to make contact with, and use of, results already available in the literature. The scheme (which includes all cases found so far in practice) runs as follows: One assumes that the variables $g_{i k}, \pi^{i k}$ can be separated into two sets, $\left(\varphi^{x}(x), \pi_{\alpha}(x)\right)$ and $\left(\psi^{\mathrm{A}}(x), \pi_{\mathrm{A}}(x)\right)$, by a bijective, time-independent, functionally differentiable canonical transformation in such a way that

(a) the surface integral $(7.56)$ depends only on the $\varphi^{x}$ and the $\pi_{\alpha}$

(b) when the $\pi_{\alpha}$ are prescribed as functions $p_{\alpha}$ of $x$ in such a way that ${ }^{(*)}$

$$
\dot{p}_{\alpha}=\mathrm{o},
$$

(*) After coordinate conditions are imposed the deformation of the hypersurface is globally determined by $\alpha^{\prime \mu}$ and $\beta^{\mu \nu}$ (the freedom of making arbitrary deformations in the interior has disappeared). It then becomes meaningful to ask for the generator of such a deformation. The generator is precisely the surface integral $(7.56)$. This is the geometrical reason why (7.56) acquires well-defined Poisson brackets after the gauge has been fixed.

(**) Strictly speaking Eq. $(7.57 a)$ is the weak equation $\pi_{\alpha}(x, t)-p_{\alpha}(x) \approx 0$. However in the following we shall not insist on the weak equality symbol unless confusion could arise. 
the constraints $\mathscr{H}_{1} \approx 0, \mathscr{H}_{i} \approx 0$ can be solved to cxpress the $\varphi^{x}$ as functionals $(7.576)$

$$
\varphi^{x}=f^{x}\left[\psi^{\mathrm{A}} ; \pi_{\mathrm{A}}\right]
$$

of the remaining canonical variables. The functional derivatives of $f^{x}$ with respect to $\psi^{A}, \pi_{\mathrm{A}}$ are assumed to exist.

If the above conditions are true, then Hamilton's equations for the Hamiltonian

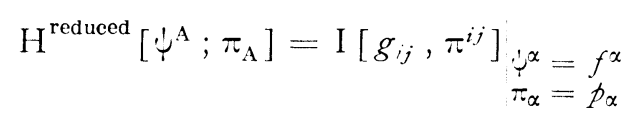

together with Eqs. (5.57) are equivalent to Einstein's equations in the particular frame defined by $\pi_{x}=p_{x}$.

Proof. Recalling that Poisson brackets are invariant under canonical transformations and that the Hamiltonian is unchanged if the canonical transformation is independent of time, we have

$$
\begin{aligned}
\dot{H}^{\mathrm{A}}(x)=\frac{\delta \mathrm{H}}{\delta \pi_{\mathrm{A}}(x)} & \\
& \begin{array}{l}
\dot{\alpha}=f^{\alpha} \\
\pi_{\alpha}=p_{\alpha}
\end{array}
\end{aligned}
$$

On the other hand

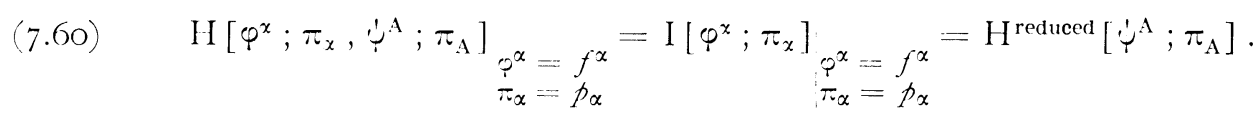

Next, differentiating (7.60) with respect to $\pi_{\mathrm{A}}$ we get

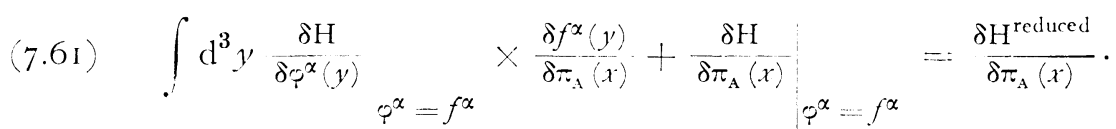

$$
\begin{aligned}
& \begin{array}{ll}
\hat{q}^{\alpha}=f^{\alpha} & \hat{p}^{\alpha}=f^{\alpha} \\
\pi_{\alpha}=p_{\alpha} & \pi_{\alpha}=p_{\alpha}
\end{array}
\end{aligned}
$$

However, by Eq. $(7 \cdot 57 a)$

$$
\begin{aligned}
& \dot{\pi}_{x}(x)=-\frac{\delta \mathrm{H}}{\delta_{\rho^{\alpha}}(y)} \quad=0
\end{aligned}
$$

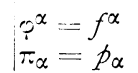

whence

$$
\frac{\frac{\delta \mathrm{H}}{\delta \pi_{\mathrm{A}}(x)}}{}=\frac{\delta \mathrm{H}^{\text {reduced }}}{\delta \pi_{\mathrm{A}}(x)}
$$

Equation (7.62) inserted back into (7.59) shows that $\mathrm{H}^{\text {reduced }}$ generates the correct equation of motion for ${ }^{\mathrm{A}}$. In a completely analogous way one shows that the correct equation of motion is also obtained for $\pi_{\mathrm{A}}$. The evolution of $\varphi^{x}$ as calculated from $(7.57 b)$ will agree with the one given by the unreduced formalism because the constraints are preserved in time. 
Note that we have not allowed for an explicit dependence of $I$ on $\dot{\psi}^{\wedge}, \pi_{A}$ i.e. a dependence other than the one induced by $\varphi^{x}=f^{x}$ ) because such a dependence would in general cause the variational derivatives $\delta \mathrm{H}^{\text {reduced }} / \delta u^{\prime \prime}$, $\delta \mathrm{H}^{\text {redured }} / \delta \pi_{\mathrm{A}}$ to be undefined. This follows from the fact that $\mathrm{H}_{0}$ and $\mathrm{I}$ do not separately have well- defined functional derivatives, only the sum $\mathrm{H}_{0}+\mathrm{I}$ does. For an explicit example of the issue discussed here see the discussion of (7.109) below.

In practice, the new phase space coordinates $\left(\varphi^{\alpha} ; \pi_{\alpha}\right),\left(\psi_{1}^{\prime} ; \pi^{4}\right)$ are not canonical. Nevertheless, one can easily verify along the same lines as the above proof that $\mathrm{H}^{\text {reduced }}$ is still obtained by inserting the solution of the constraints into $\mathrm{I}\left[g_{i j}, \pi^{i j}\right]$ provided that the following restrictions hold:

$$
\text { (a) } \begin{aligned}
\left\{\varphi^{x}(x), \dot{u}^{\mathrm{A}}\left(x^{\prime}\right)\right\} & =\left\{\varphi^{x}(x), \pi_{1}\left(x^{\prime}\right)\right\}=\left\{\pi_{x}(x), \dot{\varphi}^{A}\left(x^{\prime}\right)\right\}= \\
& =\left\{\pi_{x}(x), \pi_{1}\left(x^{\prime}\right)\right\}=\left\{\pi_{x}(x), \pi_{i}\left(x^{\prime}\right)\right\}=0 .
\end{aligned}
$$

(1)) The "matrix" $\left\{\pi_{x}(x), \varphi^{\prime 3}\left(x^{\prime}\right)\right\}$ is invertible, i.e.

$$
\int \mathrm{d}^{3} x^{\prime}\left\{\pi_{x}(x), p^{\beta}\left(x^{\prime}\right)\right\} f_{\beta}\left(x^{\prime}\right)=0 \Rightarrow f_{\beta}=0 .
$$

If equations (7.63) and (7.64) hold, then $\mathrm{H}^{\text {reduced }}$ given 1$) \mathrm{y}(7.58)$ will generate the correct equations of motion

$$
\dot{u}^{\prime}=\left\{\dot{u}^{\prime}, \mathrm{H}^{\text {reduced }}\right\} \quad, \quad \dot{\pi}_{\mathrm{A}}=\left\{\pi_{\mathrm{A}}, \mathrm{H}^{\text {redincel }}\right\}
$$

provided that the Poisson brackets are computed using the general formulat

$$
\begin{aligned}
& \{\mathrm{F}, \mathrm{G}\}=\left\{\mathrm{Q}^{a}, \mathrm{Q}^{b}\right\} \begin{array}{ll}
\partial \mathrm{F} & \mathrm{a}(\mathrm{i} \\
\partial \mathrm{Q}^{a} & \partial \mathrm{Q}^{b}
\end{array}-\left\{\mathrm{P}_{a}, \mathrm{P}_{b}\right\} \frac{\partial \mathrm{F}}{\partial \mathrm{P}_{a i}} \partial \mathrm{P}_{b} \\
& +\left\{Q^{a}, \mathrm{P}_{b}\right\}\left(\begin{array}{cc}
\partial \mathrm{F} & \partial \mathrm{C}_{i} \\
\partial \mathrm{Q}^{a} & \partial \mathrm{P}_{b}
\end{array}-\begin{array}{cc}
\partial \mathrm{i} & \partial \mathrm{F} \\
\partial \mathrm{Q}^{a} & \partial \mathrm{P}_{b}
\end{array}\right)
\end{aligned}
$$

which holds for a general (not necessarily canonical) set of phase space coordinates $\left(\mathrm{Q}^{a}, \mathrm{P}_{a}\right)$.

We now proceed to apply the above procedure to the gauges that have been proposed in the literature: the Arnowitt, Deser and Misner (I962) "T- T gauge" (where "T-T" stands for "transverse-traceless") and the so called " maximal-slicing" gauge condition proposed by Dirac (1958c).

(a) ADM's " $T-T$ " Gauge.

Arnowitt, Deser and Misner (1962) separated the canonical variables into two sets as follows: (i) $\left(g^{\mathrm{T}} ; \pi^{i} ; \pi^{\mathrm{T}} ; g_{i}\right)$ and (ii) two (independent) pairs $\left(g_{i j}^{\mathrm{TT}} ; \pi^{i j \mathrm{TT}}\right)$. The new variables are defined by applying to both $g_{i, j}-\delta_{i j}$ and $\pi^{i j}$ the decomposition.

$$
\begin{aligned}
& f_{i j}=f_{i j}^{\mathrm{TT}}+f_{i j}^{\mathrm{T}}+f_{i, j}+f_{j, i} \\
& f_{i}=\frac{\mathrm{I}}{\Gamma^{2}}\left[f_{i \jmath, j}-\frac{\mathrm{I}}{2 \Gamma^{2}} f_{k j, k i j}\right],
\end{aligned}
$$




$$
f_{i j}^{\mathrm{T}}=\frac{\mathrm{I}}{2}\left[\delta_{i j} f^{\mathrm{T}}-\underset{\Gamma^{2}}{\mathrm{I}} f_{, i j}^{\mathrm{T}}\right]
$$

and

$(7.70)$

$$
f^{T}=f_{i i}-{ }_{\Gamma^{2}}^{\mathrm{I}} f_{i, i, i j} \text {. }
$$

Here $\mathrm{I} / \nabla^{-2}$ is the inverse of the flat space Laplacian, with appropriate boundary conditions at infinity. The fixation of coordinates is achieved by imposing

$$
\begin{aligned}
& \pi^{\mathrm{T}}=\mathrm{o} \\
& g_{i}=x^{i}
\end{aligned}
$$

Thus $g^{\mathrm{T}}$ and $\pi^{i}$ correspond to the $\varphi^{\alpha}$ of Eq. (7.63) and $\pi^{\mathrm{T}}$ and $g_{i}$ to the $\pi_{x}$. The role of $\left(\mu^{\mathrm{A}} ; \pi_{\mathrm{A}}\right)$ is then played $\mathrm{by} g_{i j}^{\mathrm{TT}}$ and $\pi^{i j^{\mathrm{T} T} \text {. }}$

The Poisson brackets of the new variables are

$$
\begin{aligned}
& \left\{g^{\mathrm{T}}(x), \pi^{\mathrm{T}}\left(x^{\prime}\right)\right\}=2 \delta\left(x, x^{\prime}\right), \\
& \left\{\pi^{i}(x), g_{k}\left(x^{\prime}\right)\right\}=\frac{\mathrm{I}}{2 \Gamma^{2}}\left(\delta_{i k}-\frac{\mathrm{I}}{2 \Gamma^{2}} \dot{ }_{i} \vartheta_{k}\right) \delta\left(x, x^{\prime}\right) \\
& \left\{g_{i j}^{\mathrm{TT}}(x), \pi^{k l \mathrm{TT}}\left(x^{\prime}\right)\right\}=\delta_{i j}^{\mathrm{TT} k l} \delta\left(x, x^{\prime}\right),
\end{aligned}
$$

all others being zero.

The symbol $\delta_{i j}^{\mathrm{TT} T k l}$ in (7.75) represents the integro-differential operator which projects a symmetric tensor onto its "T-T" part, namely,

$$
\begin{aligned}
& \delta_{i j}^{\operatorname{TT} k l}-\frac{1}{2}\left[\left(\delta_{i l}-\frac{\partial_{i} \hat{v}_{l}}{\Gamma^{2}}\right)\left(\delta_{j k}-\begin{array}{c}
\partial_{j} \partial_{k} \\
\Gamma^{2}
\end{array}\right)+\left(\delta_{i k}-\begin{array}{c}
\partial_{i} \hat{i}_{k} \\
\Gamma^{2}
\end{array}\right)\left(\delta_{j l}-\frac{\partial_{j} \partial_{l}}{\Gamma^{2}}\right) \cdots\right. \\
& \left.-\left(\delta_{k l}-\begin{array}{c}
\partial_{k} \partial_{l} \\
\nabla^{2}
\end{array}\right)\left(\delta_{i j}-\frac{\partial_{i} \partial_{j}}{\Gamma^{2}}\right)\right] \text {. }
\end{aligned}
$$

The operator $(7 \cdot 76)$ satisfies the following relations

$$
\delta_{i j}^{\mathrm{TT} i j}=2 \text {, }
$$

$$
\begin{aligned}
& \delta_{i j}^{\mathrm{TT} k l} \delta_{k i}^{\mathrm{TT} m n}=\delta_{i j}^{\mathrm{TT} m,}, \\
& \delta_{j i}^{\mathrm{TT} \mathrm{T} k l} f^{\mathrm{TT} T i j}=f^{\mathrm{TT} k l}, \\
& \delta_{i j}^{\mathrm{TT} k l}=\delta_{k l}^{\mathrm{TTT} i j}, \\
& \delta_{i i}^{\mathrm{TT} k l}=2_{i} \delta_{i m l}^{\mathrm{TT} \mathrm{T} k l}=0 .
\end{aligned}
$$

Equation (7.77) expresses the fact that there are only two independent canonical pairs $\left(g_{i j}^{\mathrm{TT}} ; \pi^{k / \mathrm{TT}}\right)$ per space point. From Eqs. $(7.73)-(7.75)$ one easily checks that conditions $(7.63)$ and 7.64$)$ are satisfied. [The only result that is not immediately obvious is

$$
\Gamma^{\mathrm{I}}\left(\delta_{i k}-\underset{2 \Gamma^{2}}{\mathrm{I}} \hat{i}_{i} \hat{i}_{k}\right) f^{k}=\mathrm{O} \Rightarrow f^{k}=\mathrm{O} .
$$

This is easily checked by making appropriate contractions and recalling that, with zero boundary conditions at infinity, $\left.\left(1 / \Gamma^{2}\right)(0)=0\right]$. 
Next, let us write down more explicitly the reduced Hamiltonian corresponding to this gauge. Once gauge conditions have been imposed, the part of the surface integral $(7 \cdot 56)$ obtained by setting $\alpha^{i}=\beta_{r}^{\mu}=0$ and $\alpha^{1}=\mathrm{I}$, namely $\mathrm{P}^{1}$, is what one usually calls "the Hamiltonian". We now write down $\mathrm{P}^{\perp}$ and leave $\mathrm{P}^{i}$ and $\mathrm{M}_{\mu \nu}$ to the reader. In the new variables the surface integral defining $\mathrm{P}^{\perp}$ is

$$
\mathrm{P}^{1}\left[g_{i j}, \pi^{i j}\right]:: \oint \mathrm{d}^{2} s_{k}\left(g_{i k, i}-g_{i i, k}\right)=-\oint \mathrm{d}^{2} s_{k} g^{\mathrm{T}}, k .
$$

Following Arnowitt, Deser and Misner (1962), we assume that, when conditions (7.71) and (7.72) hold, the constraints can be solved to give

$$
\begin{aligned}
& g^{\mathrm{T}}=f^{\mathrm{T}}\left[g_{i j}^{\mathrm{TT}}, \pi^{k l \mathrm{TT}}\right], \\
& \pi^{i}=f_{i}\left[g_{i j}^{\mathrm{TT}}, \pi^{k l \mathrm{TT}}\right] .
\end{aligned}
$$

Then the reduced generator of pure time translations at infinity is

$$
\mathrm{P} 1 \text { reduced }=-\oint \mathrm{d}^{2} s_{k} g_{, k}^{\mathrm{T}}\left[g_{i j}^{\mathrm{TT}}, \pi^{k l \mathrm{TT}}\right]
$$

The rate of change of the dynamical variables under a surface deformation which is asymptotically a pure time translation is

$$
\begin{aligned}
& \dot{g}_{i j}^{\mathrm{TT}}=\left\{g_{i j}^{\mathrm{TT}}, \mathrm{P}^{1 \text { reduced }}\right\}=\delta_{i j}^{\mathrm{TT} k l} \frac{\delta \mathrm{P}^{1 \text { reduced }}}{\delta \pi^{k l \mathrm{TT}}}- \\
& \dot{\pi}^{k l \mathrm{TT}}=\left\{\pi^{k l \mathrm{TT}}, \mathrm{P}^{\perp \text { reduced }}\right\}=-\delta_{i j}^{\mathrm{TT} k l} \frac{\delta \mathrm{P}^{\perp \text { reduced }}}{\delta g_{i j}^{\mathrm{TT}}}
\end{aligned}
$$

with $\delta_{i j}^{\mathrm{TT} k l}$ and $\mathrm{P}^{\mathrm{L} \text { reduced }}$ given by $(7.76)$ and (7.84) respectively. The presence of the "projection operator" $\delta_{i j}^{\mathrm{Tr} k l}$ in $(7.85)$ and (786.) ensures that the right hand sides of those equations are transverse and traceless. Equations totally similar to $\left(7.85^{-86}\right)$ hold of course for the changes generated by $\mathrm{P}^{i \text { reduced }}$ and $\mathrm{M}_{\rho \sigma}^{\text {reduced }}$.

Finally, let us evaluate the Dirac brackets of the original variables $g_{i j}$, $\pi^{k l}$ in the gauge $(7.7 \mathrm{I}-72)$. The original definition (1.58) of the Dirac bracket implies that, in the phase space coordinates $\left(g^{\mathrm{T}}, \pi^{\mathrm{T}}\right),\left(g_{i}, \pi^{l}\right),\left(g_{i j}^{\mathrm{TT}}, \pi^{k l \mathrm{TT}}\right)$, the bracket is obtained simply by dropping the terms involving $\left(g^{\mathrm{T}}, \pi^{\mathrm{T}}\right)$ and $\left(g_{i}, \pi^{l}\right)$ from the original Poisson bracket (7.66). Simultaneously, one should consider the second class constraints $(7.71),(7.72)$ and (7.83) as strong equations. This implies that the Dirac bracket between the T-T variables is given just by (7.75). To find the Dirac brackets of the original variables, one then returns to the decomposition (7.67) and proceeds as follows: After taking (7.71), (7.72) and (7.83) to be strong equations one has from (7.67):

$$
\begin{aligned}
& g_{i j}=g_{i j}^{\mathrm{TT}}+f_{i j}^{\mathrm{T}}+2 \delta_{i j}, \\
& \pi^{k l}=\pi^{k l \mathrm{TT}}-f_{k, l}+f_{l, k} \equiv \equiv \pi^{k l \mathrm{TT}}+f_{(l, k)}
\end{aligned}
$$


where $f_{i j}^{\mathrm{T}}$ is related to $f^{\mathrm{T}}$ by $(7.69)$. This gives, for example,

$$
\begin{gathered}
\left\{g_{i j}(x), g_{m n}\left(x^{\prime}\right)\right\}^{*}=\left\{g_{i j}^{\mathrm{TT}}(x), f_{m n}^{\mathrm{T}}\left(x^{\prime}\right)\right\}^{*}- \\
\left.-\ldots g_{m n}^{\mathrm{TT}}\left(x^{\prime}\right), f_{i j}^{\mathrm{T}}(x)\right\}^{*}+\left\{f_{i j}^{\mathrm{T}}(x), f_{m n}^{\mathrm{T}}\left(x^{\prime}\right)\right\}^{*}
\end{gathered}
$$

where

$$
\left\{g_{i j}^{\mathrm{TT}}(x), f_{m n}^{\mathrm{T}}\left(x^{\prime}\right)\right\}^{*}=\delta_{i j}^{\mathrm{TT} a b}(x) \frac{\delta f_{m n}^{\mathrm{T}}\left(x^{\prime}\right)}{\delta \pi^{a b \mathrm{TT}}(x)}
$$

and

$$
\begin{gathered}
\left\{f_{i j}^{\mathrm{T}}(x), f_{m n}^{\mathrm{T}}\left(x^{\prime}\right)\right\}^{*}=\int \mathrm{d}^{3} y \delta_{c d}^{\mathrm{TT} a b}(y) \times \\
\times\left[\frac{\delta f_{i j}^{\mathrm{T}}(x)}{\delta g_{c d}^{\mathrm{TT}}(y)} \frac{\delta f_{m n}^{\mathrm{T}}\left(x^{\prime}\right)}{\delta \pi^{a b \mathrm{~T}^{\mathrm{T} T}}(y)}-\frac{\delta f_{m u}^{\mathrm{T}}\left(x^{\prime}\right)}{\delta g_{c d}^{\mathrm{TT}}(y)} \frac{\delta f_{i j}^{\mathrm{T}}(x)}{\delta \pi^{a b \mathrm{~T} \mathrm{~T}}\left(y^{\prime}\right)}\right] .
\end{gathered}
$$

Equations (7.89)-(7.91) express the Dirac brackets of the $g_{i j}$ with themselves in terms of the functionals $f^{\mathrm{T}}$ defined by $(7.83)$. The remaining Dirac brackets, which will be $k \mathrm{ft}$ to the reader, contain also the functional $f_{i}$. The functionals $f^{\text {T }}$ and $f_{i}$ may be obtained in practice to any desired accuracy by the method of successive approximations. The T-T quantities in the right hand side of (7.89)-(7.91) are to be expressed in terms of the $g_{i j}, \pi^{k l}$ by means of the projection operator (7.76). The equations of motion $(7.85)-(7.86)$ can be rewritten in terms of the bracket as

$$
\dot{\mathrm{F}}=\{\mathrm{F}, \mathrm{H}\}^{*}
$$

where $\mathrm{H}$ is given by $(7.56)$ and $\mathrm{F}$ is an arbitrary functional of the $g_{i j}, \pi^{k l}$. It is understood that in evaluating the right hand side of $(7.92)$ one takes the surface integral appearing in $\mathrm{H}$ outside of the bracket and performs the integration at the end.

(b) Dirac's "Maximal Slicing" Gauge

The one parameter family of surfaces on which the evolution is being observed may be fixed by the " maximal slicing" condition $\left(^{*}\right)$

$$
\pi=\pi_{i}^{i} \approx 0 \text {. }
$$

$\left({ }^{*}\right)$ Equation (7.93) is called the "maximal slicing condition", because on account of (7.29) it can be equivalently written as

$$
\mathrm{K}=\mathrm{o}
$$

where $\mathrm{K}$ is the trace of the extrinsic curvature tensor. Now the change under a deformation $\mathrm{N}^{\mu} \mathrm{d} t$ of the volume $\Delta \mathrm{V}=\int \mathrm{d}^{3} x g^{1 / 2}$ enclosed in a three dimensional region is

$$
\mathrm{d}(\Delta \mathrm{V})=\mathrm{d} \dot{\int}\left(g^{1 / 2}\right) \mathrm{d}^{3} x=-j \mathrm{~N}^{1} g^{1 / 2} \mathrm{Kd}^{3} x,
$$

by (B.5). This shows that if $K=0$, the volume of the three-surface is unchanged by a surface deformation. It may be shown that for a spacelike hypersurface embedded on a hyperbolic Riemannian space one is actually dealing with a maximum. 
Condition (7.93) is invariant under changes of coordinates in the surface and so does not restrict the spatial coordinate system. The Hamiltonian will therefore be only partially reduced by condition (7.93). It will still contain a term $\int N^{i} \mathscr{H}_{i}$ and will still possess the three constraints $\mathscr{H}_{i} \approx$ o corresponding to the freedom to make arbitrary tangential cleformations in the surface. To achicve total reduction one can still impose three coordinate conditions, but we shall not worry about that here (see Dirac, $1958 \mathrm{c}$ ).

As I)irac ( $1958 \mathrm{C}$ ) realized, one may rearrange the canonical variables in the following way: (a) One pair $(\varphi ; \pi)$ and (b) Five other pairs $\left(\bar{g}_{i j} ; \tilde{\pi}^{i j}\right)$. The quantities under consideration are defined as follows:

$\begin{array}{ll}(7.94) & \varphi=\frac{1}{3} \log g, \\ (7.95) & \pi=g_{i, j} \pi^{i j}, \\ (7.96) & \tilde{g}_{i, j}=g^{1 / 3} g_{i, 1} \\ (7.97) & \tilde{\pi}^{i j}=g^{1,3}\left(\pi^{i, j}-\frac{\mathrm{I}}{3} \pi g^{i j}\right) .\end{array}$

They have the following Poisson brackets:

$$
\begin{array}{lc}
\{.08 & \left\{\varphi(x), \pi\left(x^{\prime}\right)\right\}=\delta\left(x, x^{\prime}\right) \\
(7.90) & \left\{\bar{g}_{i, j}(x), \pi^{k}\left(x^{\prime}\right)\right\}=\delta_{i j}^{k l} \delta\left(x, x^{\prime}\right)
\end{array}
$$

with

$$
\tilde{\delta}_{l, j}^{k l}=\frac{\mathrm{I}}{2}\left(\delta_{i}^{k} \delta_{j}^{l}+\delta_{i}^{l} \delta_{j}^{k}\right)-\frac{\mathrm{I}}{3} \tilde{g}_{i j} \tilde{g}^{k l}
$$

and

$$
\left\{\tilde{\pi}^{i j}(x), \tilde{\pi}^{k l}\left(x^{\prime}\right)\right\}=\frac{1}{3}\left(\tilde{\pi}^{i j} \tilde{y}^{l l}-\tilde{\pi}^{k l} \tilde{y}^{i j}\right) \delta\left(x, x^{\prime}\right)
$$

All other Poisson brackets are zero.

The quantity $g^{i j}$ appearing in $(7.96)$ and $(7.97)$ is the inverse of the conformal metric $\tilde{g}_{i, j}$, i.e. $\bar{g}^{l m} \bar{g}_{l m}=\delta_{s}^{l}$, and is related to the full metric by $\bar{g}^{i j}=g^{1 / 3} g^{i, j}$. The " conformal Kronecker delta" defined by (7.IOO) has the following useful properties:

$$
\begin{array}{ll}
(7.102) & \tilde{\delta}_{i j}^{i j}=5, \\
(7.103) & \tilde{\delta}_{k l}^{i j} \tilde{\delta}_{m n}^{k l}=\bar{\delta}_{m n}^{i j}, \\
(7.104) & \tilde{\delta}_{l, j}^{k l} \tilde{g}_{k l}=\tilde{\delta}_{i j}^{k l} \bar{r}^{i j}=0 \\
(7.105) & \tilde{\delta}_{i j}^{k l} \tau^{i j}=\bar{\pi}^{k l} .
\end{array}
$$


Equation (7.102) says that there are only five independent canonical pairs $\left(\tilde{g}_{i, j}, \tilde{\pi}^{i j}\right)$ per space point, in accordance with the conditions

(7.106)

$(7.107)$

$$
\begin{gathered}
\tilde{g}=\operatorname{det}\left\|\tilde{g}_{i j}\right\|=\mathrm{I}, \\
\pi=\tilde{g}_{i j} \tilde{\pi}^{i j}=\mathrm{O} .
\end{gathered}
$$

From Eqs. ( 7.8 ) (7.IOI) we see immediately that the new phase space coordinates satisfy the conditions $(7.63),(7.64)$ of the reduction theorem. According to Eqs. (7.66) and (7.98)-(7.101) the Poisson bracket is computed in the new variables using the equation

$$
\begin{aligned}
& \{\mathrm{F}, \mathrm{G}\}=\int \mathrm{d}^{3} x\left[\begin{array}{llll}
\delta \mathrm{F} & \delta \mathrm{C} & \delta(i & \delta \mathrm{F} \\
\delta \mathrm{c} & \delta \pi & \delta \mathrm{j} & \delta \pi
\end{array}\right.
\end{aligned}
$$

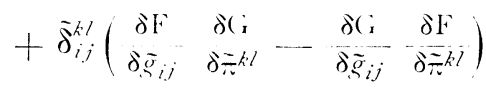

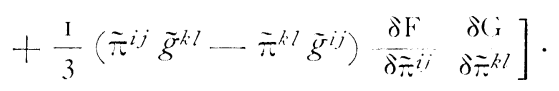

One can check from (7.108) that $\tilde{g}$ and $\tilde{\pi}$ have zero Poisson brackets with everything and, consequently, they can be set strongly equal to unity and zero respectively. Thus one can preserve the symmetry in all indices even though one is dealing with more variables than needed [remember Eqs. (7.106) and (7.107)].

In order to reduce the Hamiltonian, we have to express the surface integral $(7.56)$ as a functional of the the new variables. Again we will just work out $\mathrm{P}^{1}$ and leave $\mathrm{P}^{i}$ and $\mathrm{M}_{\sigma o}$ as an exercise for the reader. One gets:

$$
(7 . \mathrm{IOg}) \quad \mathrm{P}^{\perp}: \int \mathrm{d}^{2} s_{k}\left(g_{i k, i}-g_{i i, k}\right)=\int \mathrm{d}^{2} s_{k}\left(\tilde{g}_{i k, i}-2 g^{1: 3}, k\right)
$$

From (7.IO9) we see that to have well-defined variational derivatives of $\mathrm{P}^{1}$ reduced with respect to $\tilde{s}_{i k}$, we have to get rid of the $\tilde{g}_{i k, i}$ term. In other words, the reduction of the Hamiltonian by means of the maximal slicing condition $\pi=0$ is not possible unless one chooses a more restrictive asymptotic spatial coordinate condition than in $(7 .+0 b)$; we shall require

$$
\text { (7.IIO) } \quad \tilde{g}_{i k, i} \sim r^{(2: \varepsilon)} \text {. }
$$

The asymptotic form $(7.39)$ is thus not allowed in this context, lut the line element

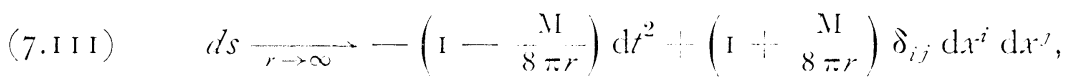

which is oltained from $(7.39)$ by a change of coordinates

$$
x_{r \rightarrow \infty}^{-i} \rightarrow x^{i}\left(\mathrm{I}-\frac{\mathrm{M}}{16 \pi r}\right)
$$

satisfies (7. I IO). 
Taking into account (7.IIO), the energy becomes

$$
\mathrm{P}^{1}=-2 \oint \mathrm{d}^{2} s_{k} g^{1 / 3}, k \text {. }
$$

One may check that, when inserted into (7.II3) the metric (7.III) correctly gives $\mathrm{P}^{1}=\mathrm{M}$. The final step in the reduction procedure is to solve the Hamiltonian constraint $\mathscr{H}_{1}=0$ and express $g^{1 / 3}$ in (7.II3) as a functional of the $\tilde{g}_{i j}$ and $\tilde{\pi}^{i j}$. This leads to the following equation, first examined by Lichnerowicz (I944) and recently extensively studied by Choquet-Bruhat (I972, 1973) and by O' Murchadha and York (1972, 1973):

$$
\tilde{\Delta} \Phi+{ }_{8}^{\mathrm{I}} \tilde{\pi}_{i j} \tilde{\pi}^{i j} \Phi^{-7}-\frac{\mathrm{I}}{8} \tilde{\mathrm{R}} \Phi=\mathrm{O}
$$

with $\Phi=g^{1 / 12}$. (Here $\tilde{\pi}_{i j}=\vec{g}_{i l} \vec{g}_{j k} \tilde{\pi}^{l k}$ and $\overrightarrow{\mathrm{R}}, \vec{\Delta}$ are, respectively, the curvature and the Laplacian in the metric $\tilde{g}_{i, j}$ ). The reduced Hamiltonian is therefore given by

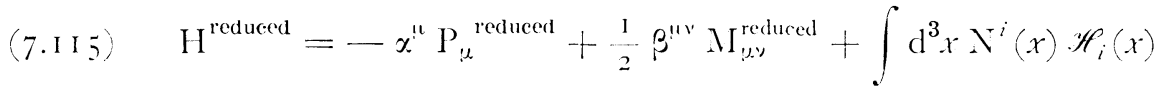

with $\mathrm{P}^{\text {Lreduced }}$ given by (7.I I3). Similar expressions hold for $\mathrm{P}^{i}$ and $\mathrm{M}_{f \sigma}$. The constraints

$$
\mathscr{H}_{i} \approx \mathrm{O}
$$

still hold as first class equations. The tangential generator appearing in ( 7 . I 15 ), (7.116) is also understood to be reduced by the condition $\pi=0$. It may be written in the form

$$
\mathscr{H}_{i}=-2 \tilde{\pi}_{i \mid . j}^{j}
$$

with the covariant derivative being taken in the metric $\tilde{g}_{i j}$.

Finally we write the equations of motion for $\tilde{g}_{i j}$ and $\tilde{\pi}^{i j}$ in a more explicit form. From (7.108) these equations are seen to read

$$
\begin{gathered}
\dot{\tilde{g}}_{i j}=\left\{\tilde{g}_{i j}, \mathrm{H}^{\text {reduced }}\right\}=\tilde{\delta}_{i j}^{k l} \frac{\delta \mathrm{H}^{\text {reduced }}}{\delta \tilde{\pi}^{k l}} \\
\dot{\tilde{\pi}}^{k l}=\left\{\tilde{\pi}^{k l}, \mathrm{H}^{\text {reduced }}\right\}=-\tilde{\delta}_{i j}^{k l} \frac{\delta \mathrm{H}^{\text {reduced }}}{\delta \tilde{g}_{i j}}+ \\
\quad+\frac{\mathrm{I}}{3}\left(\tilde{\pi}^{k l} \tilde{g}^{i j}-\tilde{\pi}^{i j} \tilde{g}^{k l}\right) \frac{\delta \mathrm{H}^{\text {reduced }}}{\delta \tilde{\pi}^{i j}}
\end{gathered}
$$

with $\tilde{\delta}_{i j}^{k l}$ and $\mathrm{H}^{\text {reduced }}$ given by (7.100) and (7.1 I 5) respectively.

One may obtain the Dirac bracket corresponding to the gauge condition (7.93) in a way totally analogous to the one followed for the T-T gauge. The situation is simpler here because the gauge condition $\pi=0$ is just an algebraic equation. The Iirac bracket then turns out to depend on the solution of Eq. (7.II4), which as we said above has been examined in detail in the literature. 
One thus considers (7.93) and (7.II-4) as strong equations and writes the I)irac bracket as

(7.I $20 a)$

where

$(7.1206)$

and

$(7.120 c)$

$$
\begin{aligned}
\left\{g_{i j}(x), g_{k l}\left(x^{\prime}\right)\right\}^{*} & =\Phi^{4}(x)\left\{\tilde{g}_{i j}(x), \Phi^{4}\left(x^{\prime}\right)\right\}^{*} \tilde{g}_{k l}\left(x^{\prime}\right) \cdots \\
& \cdots-\Phi^{4}\left(x^{\prime}\right)\left\{\tilde{g}_{k l}\left(x^{\prime}\right), \Phi^{4}(x)\right\}^{*} \tilde{g}_{i, j}(x)+ \\
& +\tilde{g}_{i, j}(x) \tilde{g}_{k l}\left(x^{\prime}\right)\left\{\Phi^{4}(x), \Phi^{4}\left(x^{\prime}\right)\right\}^{*}
\end{aligned}
$$

$$
\left\{\tilde{g}_{i j}(x), \Phi^{1}\left(x^{\prime}\right)\right\}^{*}=\bar{\delta}_{i, j}^{m n}(x) \frac{\delta \Phi^{4}\left(x^{\prime}\right)}{\delta \tau^{m n}(x)}
$$

$$
\begin{aligned}
& \left\{\Phi^{4}(x), \Phi^{4}\left(x^{\prime}\right)\right\}^{*}=
\end{aligned}
$$

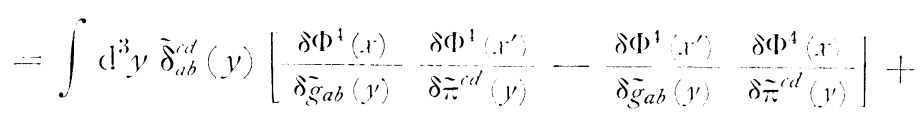

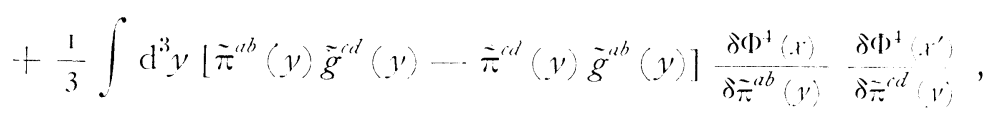

where E.qs. (7.99)-(7.IOI) and (7.108) have been used. (The righthand side of (7.120) may be expressed as a functional of the original variables $g_{i, j}, \pi^{i j}$ by means of the equations $\tilde{g}_{i j}=g^{1 / 3} g_{i, j}$ and $\tilde{\pi}^{i j}=g^{1 / 3} \pi^{i j}$. The other Dirac brackets are obtained in a similar manner and will be left to the reader. The equations of motion (7.118), (7.1 19) then read, in terms of the original variables,

$$
\dot{g}_{i j}=\left\{g_{i, j}, \mathrm{H}\right\}^{*} \quad, \quad \dot{\pi}^{i j}=\left\{\pi^{i j}, \mathrm{H}\right\}^{*}
$$

with $\mathrm{H}$ given by (7.115). Again in (7.121) as in (7.92), one deals with the surface term in $\mathrm{H}$ by taking the surface integral outside of the Poisson bracket. The constraints (7.1I6) remain first class in the starred bracket.

\section{CLOSED SPACES: York's Gange.}

When the three-surface on which the state is defined is compart, all information about the "location" of the surface in the enveloping spacetime: is contained in the $\left(g_{i j}, \pi^{i j}\right)$. There is in this case no asymptotic region and no set of asymptotic surface variables $a^{4}, b_{r}{ }_{r}$; consequently no surface integral ever enters into the Hamiltonian, which reads simply

$$
\mathrm{H}_{0}=\int \mathrm{d}^{3} x\left(\mathrm{~N}^{1} \mathscr{H}_{1} \ldots \mathrm{N}^{i} \mathscr{H}_{i}\right)
$$

Also, there are no problems now with $\mathrm{H}_{0}$ not having well-defined variational derivatives.

It is clear that the procedure used for fixing the gauge in the open case will not work for the compact case since there are no surface integrals to deal with. Moreover, a gauge condition such as $\pi=0$ will not be a satisfactory 
contlition in this case as it would lead to no dynamics at all ${ }^{(*)}$. To eliminate the freedom to make arlitrary deformations of the surface, one must use second-class constraints which are explicitly time-dependent. However, there is no general scheme available for deriving the reduced Hamiltonian in this case. One knows only that when the gauge condition is of the form $q^{0} \approx$ : then the reduced Hamiltonian is $-p_{0}$ a smay be verified by observing that the right equations of motion are obtained.

Fortunately, the only gauge condition so far proposed for compact spaces is of the simple form $q^{0} \approx \tau$ and one can then carry on the reduction procedure without difficulty.

York (197I, 1972) has proposed the condition

$$
x^{0} \approx \frac{2}{3} g^{-1 / 2} \pi
$$

to fix the spacetime slicing. Note that, as happened with the $\pi \approx$ o condition for open spaces, Eq. 7.132$)$ is invariant under changes of coordinates in the surface and therefore the constraints $\mathscr{P}_{i} \approx 0$ will still remain first class. One may get rid of these constraints by imposing three more conditions besides (7.I23). We will not carry out this step) here.

In order to deal with condition (7.I23) one needs a slightly modified version of Dirac's variables (7.94)-(7.97); one uses

$$
\begin{gathered}
\mathrm{T}=\frac{2}{3} g^{-1 / 2} \pi \\
\mathrm{P}=-g^{1: 2}
\end{gathered}
$$

instead of $\varphi$ and $\pi$ given $b y(7.94)$ and $(7.95)$. The rest of the variables remain the same. One then has

$$
\left\{\mathrm{T}(x), \mathrm{P}\left(x^{\prime}\right)\right\}=\delta\left(x, x^{\prime}\right)
$$

instead of $(7.98)$ and again, all other brackets remain the same. The Poisson bracket is then given by (7.108) with $\varphi$ replaced by $T$ and $\pi$ replaced by $P$. To eliminate the extra degrees of freedom one solves the equation $\mathscr{H}_{1}=0$ and expresses $\mathrm{P}$ as a functional of the remaining canonical variables and of the time $x^{0}$ (the feasibility of this step is the main test of whether or not (7.123) is a good gauge condition). Before gauge conditions are imposed the Poisson bracket is given by (7.108) with $\rho \rightarrow T$ and $\pi \rightarrow P$. When the gauge condition

(*) The equation $\mathscr{H}_{1} \approx 0$ would become a second class constraint after the $\pi \approx 0$ condition is imposed. Now since the gauge condition is time independent, the Hamiltonian woukl be unchanged; this means that the equations of motion would be given by

$$
\dot{\mathrm{F}}=\left\{\mathrm{F}, \mathrm{H}_{0}\right\}^{*}=\left\{\mathrm{F}, \dot{\mathrm{d}} \mathrm{d}^{3} \mathrm{x} \mathrm{N}^{i} \mathscr{H}_{i}\right\}^{*},
$$

showing that there would be no dymamics left except for changes of coordinates on the surface. Thus $\pi \approx 0$ camnot be used to fix the gauge for compact spaces. 
(7.123) is enforced, one drops the P and T terms and the bracket lecomes

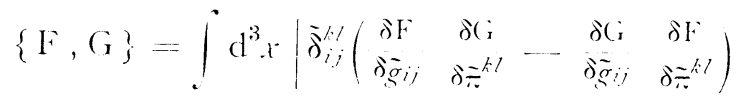

$$
\begin{aligned}
& \begin{array}{l}
1 \\
3
\end{array}\left(\bar{\pi}^{i j} \bar{g}^{k l} \ldots \bar{\pi}^{k l} \bar{g}^{i j}\right) \begin{array}{cc}
\delta \mathrm{F} & \delta(i \\
\delta \bar{\pi}^{i j} & \delta \bar{\pi}^{k l}
\end{array} \mid .
\end{aligned}
$$

The reduced Hamiltonian then reads

$$
\mathrm{H}^{\text {reduced }}=\int \mathrm{d}^{3} x g^{\frac{1}{2}} \cdots \int \mathrm{d}^{3} x N^{2} \mathscr{H}_{i} .
$$

Equation (7.128) tells us that the dynamically important part of the Hamiltonian is the volume of the surface ( $\mathscr{H}_{i}$ is connected only with changes of coordinates in the surface). In Eq. (7. I28) $g \cdot(x)$ is a functional of $g_{i j}$ and $\pi^{i j}$, and depends explicitly on the time $x^{0}$.

In order to express $g^{\frac{1}{2}}$ as a functional of the remaining variables, one rewrites the $\mathscr{H}_{1} \approx 0$ constraint in the form of a non-linear partial differential cquation:

$$
\text { (7.129) } \quad j \Phi \div \frac{1}{8} \dot{\pi}_{i j} \dot{\pi}^{i j} \Phi^{-7}-\frac{1}{8} \tilde{R} \Phi-\frac{3}{64}\left(r^{0}\right)^{2} \Phi^{5}=0
$$

which is a generalization of (7.IIt), (York, 1972; O' Murchadha and York 1972).

As we emphasized at the beginning, the constraints $\mathscr{H}_{2} \approx \mathrm{o}$ still hold as first class equations and can still be expressed in the form 7.177 . The I)irac brackets corresponding to the gauge condition (7.123) are the same as those exemplified by (7.120) corresponding to the $\pi \approx 0$ condition. The only change is that now the functional $\Phi$ appearing in (7.120) solves (7.I29), instead of (7.II 4 ). 


\section{ApPENDiX A}

\section{METRIC CONVENTIONS}

One of the most vexing problems encountered in writing about relativistic physics is the choice of a metric convention. We have chosen to use the metric $\mathrm{I}=g^{11}=g^{2 \cdot 2}=g^{33}=-g^{00}$ in the main text because the transition between nonrelativistic and relativistic canonical momenta seems more straightforward. This convention also agrees with most of the current literature on general relativity. On the other hand, it disagrees with the latest edition of Landau and Lifshitz's Classical Theory of Fields (197I) and with Bjorken and Drell (1964). We therefore hope that the following table showing the relation of our conventions to those of Landau and Lifshitz and of Bjorken and Drell will help in some small way to make this work more useful to those who prefer other metric conventions. Our conventions for general relativity agree with those of Misner, Thorne and Wheeler (1973) provided Newton's gravitational constant $G$ is set equal to $(16 \pi)^{-1}$. The reader may also find in this reference an exhaustive comparison with other conventions used in the literature.

BJORKEN ANI) DREIL

HERE

$$
\mu=\mathrm{o}, \mathrm{I}, 2,3
$$

$$
g^{\| v v}=\left[\begin{array}{rrrr}
-1 & 0 & 0 & 0 \\
0 & 1 & 0 & 0 \\
0 & 0 & 1 & 0 \\
0 & 0 & 0 & 1
\end{array}\right]
$$$$
\mathrm{P}_{\mu,}=\left(-\mathrm{P}^{0}, \mathrm{P}^{1}, \mathrm{P}^{2}, \mathrm{P}^{3}\right)
$$$$
\mathrm{P}^{\mu} \mathrm{P}_{\mu}=\mathbf{P} \cdot \mathbf{P}-\left(\mathrm{P}^{0}\right)^{2}=-\mathrm{M}^{2}
$$$$
P_{c p}^{\mu}=\frac{1}{i} \frac{\hat{v}^{2}}{\partial x_{i \mu}}
$$$$
\mathrm{d} s=\left(-\mathrm{d} x^{\prime \prime} \mathrm{d} x_{12}\right)^{1 ! 2}
$$$$
F^{\prime \prime \prime}=i^{\prime \prime} A^{\prime \prime}-i^{\prime \prime} A^{\prime \prime} \cdots A^{\prime \prime, " ~}-A^{\prime \prime}, "
$$$$
-\pi^{i}=\mathrm{F}^{10 i}=\mathrm{E}^{i}
$$$$
\frac{\mathrm{I}}{2} \varepsilon^{i j k} \mathrm{~F}^{j k}=\mathrm{B}^{i}
$$

(LANI)AL ANI IIFSHITY, IF DIFFERENT)

$$
\begin{aligned}
& \mu=0, \mathrm{I}, 2,3 \\
& g^{\prime \prime \nu}=\left[\begin{array}{rrrr}
\mathrm{I} & 0 & 0 & 0 \\
0 & -\mathrm{I} & 0 & 0 \\
0 & 0 & -\mathrm{I} & 0 \\
0 & 0 & 0 & -\mathrm{I}
\end{array}\right] \\
& \mathrm{P}_{i \mu}=\left(\mathrm{P}^{0},-\mathrm{P}^{1},-\mathrm{P}^{2},-\mathrm{P}^{3}\right) \\
& \mathrm{P}^{\prime \prime} \mathrm{P}_{\mu}=\left(\mathrm{P}^{0}\right)^{2}-\mathbf{P} \cdot \mathbf{P}=+\mathrm{M}^{2} \\
& \mathrm{P}_{p h}^{i \alpha}=i \frac{\hat{c}}{i x_{i \mu}} \\
& \mathrm{d} s=\left(+\mathrm{d} x^{\prime \prime} \mathrm{d} x_{\mu \mu}\right)^{1 / 2}
\end{aligned}
$$

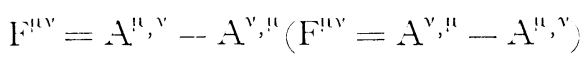

$$
\begin{aligned}
& \pi^{i}=\mathrm{F}^{(1 i}=\mathrm{E}^{i}\left(-\pi^{i}=\mathrm{F}^{(0 i}=-\mathrm{E}^{i}\right) \\
& \frac{\mathrm{I}}{2} \varepsilon^{i j k} \mathrm{~F}^{j k}=\mathrm{B}^{i}\left(\frac{\mathrm{I}}{2} \varepsilon^{i j k} \mathrm{~F}^{j k}=-\mathrm{B}^{i}\right)
\end{aligned}
$$


We have also chosen a different null-plane metric from that of Kogut and Soper (1970). Our convention has the advantage that all components of $g^{i, y}$ are positive even though we are in a Minkowski space.

HERE

$$
\begin{aligned}
& \mu=\cdots,-, I, 2 \\
& g^{\prime \prime \prime}==\left|\begin{array}{cccc}
0 & 1 & 0 & 0 \\
1 & 0 & 0 & 0 \\
0 & 0 & 1 & 0 \\
0 & 0 & 0 & 1
\end{array}\right| \\
& \mathrm{P}^{ \pm}=\begin{array}{c}
\mathrm{I} \\
12
\end{array}\left(\mathrm{P}^{3} \pm \mathrm{P}^{0}\right)=\mathrm{P}_{\mp} \\
& \mathrm{P}^{\mu \prime} \mathrm{P}_{\mu}=2 \mathrm{P}^{\mathrm{P}} \mathrm{P}^{-}+\mathbf{P}^{\prime \prime}=\ldots \mathrm{M}^{2}
\end{aligned}
$$

$$
\begin{aligned}
& \text { KOGL'T ANI) SOPER } \\
& \mu=\cdots, 1,2,- \\
& g^{\prime \prime v}=\left|\begin{array}{rrrr}
0 & 0 & 0 & \text { I } \\
0 & \cdots-1 & 0 & 0 \\
0 & 0 & -1 & 0 \\
\text { I } & 0 & 0 & 0
\end{array}\right| \\
& P^{ \pm}=\frac{1}{12}\left(P^{0}+P^{3}\right)=P_{\mp} \\
& \mathrm{P}^{\prime \prime} \mathrm{P}_{\eta}=2 \mathrm{P}^{\prime} \mathrm{P}^{-}-\mathbf{P}^{\prime 2}=: \mathrm{I}^{2}
\end{aligned}
$$

\section{APPENDIX B}

\section{Extrinsic Curvature and the Embedding Equations of Gauss and Coduzzi}

Consider a spacelike hypersurface embedded in a four-dimensional spacetime of hyperbolic signature. Let the unit normal to the surface be $n^{\text {in. }}$. Denote by the suffix $\perp$ the projection of a spacetime tensor of any rank on the normal (i.e. $A_{\perp}=A_{\mu} x^{\prime \prime}=-A^{\perp}$ and similarly for higher order tensors) and by latin indices the projection onto the surface. The following relations the hold in the basis $\left(\boldsymbol{n}, \boldsymbol{e}_{i}\right)$ where $\boldsymbol{e}_{i}$ denotes the tangent vector to the $i$-th coordinate line on the surface:

$$
\begin{gathered}
{ }^{(4)} \mathrm{R}_{a b c d}=\mathrm{R}_{a b c d}+\mathrm{K}_{a c} \mathrm{~K}_{b l d}-\mathrm{K}_{a d d} \mathrm{~K}_{b c}, \\
{ }^{(4)} \mathrm{R}_{1 b c d}=\mathrm{K}_{b d l c}-\mathrm{K}_{b i d d} .
\end{gathered}
$$

Equations (B.I) are known as the Gauss-Codazzi equations (see, for example Eisenhart, 1926).

The symmetric space tensor $\mathrm{K}_{a b}$ appearing in (B. I) is calle the extrinsic curvature or second fundamental form of the surface and it has, from the Hamiltonian point of view, the important property of being determined completely once the surface is given. The extrinsic curvature is geometrically defined by the parallel transport of the normal. $n^{i \cdot}$ along the surface. Since $n^{i .}$ has unit length, the difference $\mathrm{D} n$ between $x^{2 .-}$ at the point $x^{k}+\mathrm{d} x^{k}$ and the vector obtained by parallel transporting $n^{i .}$ from $x^{k}$ to $x^{k}+\mathrm{d} x^{k}$ along the surface lies on the surface. One then defines $\mathrm{K}_{a b}$ by writing:

$$
{ }^{(1)} \mathrm{D} n_{b}=-\mathrm{K}_{a b} \mathrm{~d} x^{a}
$$


The lefthand side of (B.2) may be expressed in terms of the lapse and shift functions as follows: First one notices from $(7.5)-(7.8)$ that

$$
\text { (B.3) } \quad n_{\mu}=\left(\cdots N^{\perp}, \mathrm{o}, \mathrm{O}, \mathrm{O}\right)
$$

The minus sign in $n_{0}$ ensures that $n^{\prime \prime}$ points in the direction of increasing $x^{0}$, Using Eqs. $(7.5)-(7.8)$ we found that $(\mathrm{B} .3)$ implies

$$
\text { (B.4) } \begin{aligned}
(1) & \mathrm{I}) n_{b}=-{ }^{(1)} \Gamma_{a b}^{\prime \prime} n_{0} \mathrm{~d} x^{b}= \\
& =\left(2 N^{1}\right)^{-1}\left[\left(-g_{a b, 0}+g_{0, a, b}+g_{0 b, a}\right)-N^{c}\left(-g_{a b, c}+g_{a, b, b}+g_{c b, a}\right)\right]= \\
& =\left(2 N^{-1}\right)^{-1}\left(-g_{a b, 0}+N_{a, b}+N_{b, a}-2 \Gamma_{a b} N_{c}\right)== \\
& =-\left(2 N^{1}\right)^{-1}\left(-g_{a b, 0}+N_{a, b}+N_{b, a}\right) .
\end{aligned}
$$

So Ne learn that

$$
\mathrm{K}_{a b}=\left(2 \mathrm{~N}^{1}\right)^{-1}\left(-g_{a h, 0)}: X_{a b}: X_{h, a}\right.
$$

which in particular shows that $\mathrm{K}_{a b}$ is symmetric.

2. Proof of Eq. (7.18)

The following relations hold in the basis $\boldsymbol{n}, \boldsymbol{e}_{a}$ ):

$$
\begin{aligned}
& g^{i \mu \sigma}=c_{a b}^{\prime \prime} e^{\sigma a}-u^{\prime \prime} u^{\sigma} \\
& \mathrm{K}_{a b}=-c_{a b} l^{\alpha} ; c_{a b} \\
& n_{i,} n^{\prime \prime}=-1 \text {, } \\
& n_{p} c_{a}^{i,}=0
\end{aligned}
$$

(B.)

Equation (B.7) is just (B.2) written in general coordinates.

From (B.6), we get

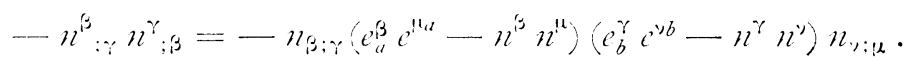

since the covariant derivative of $(B .8)$ is given by

$$
n_{\mu ; \beta} n^{\mu}=\mathrm{O}
$$

Eq. (B.IO) reduces to

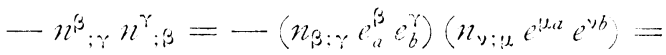

$$
\begin{aligned}
& =-\mathrm{K}_{a b} \mathrm{~K}^{a b}
\end{aligned}
$$

The last step in (B. I2) follows from (B.7). This proves (7.18a). Equation $(7.18 b)$ is proved in an entirely analogous manner.

\section{Mom:ntum and Angular Momentum of the Gravitational Fidd.}

As an illustration, let us sketch how $(7 .+86)$ is identified as the linear momentum. The linear momentum is normally defined as the conserved quantity associated with invariance of the action under translations. A tran- 
slation is an operation which is defined only for a flat space - it amounts to displacing the fields from the point $x^{k}$ to the point $x^{k}: \varepsilon^{k}$, with $\varepsilon^{k}$ fixed (independent of $x$ ) and where the $x^{k}$ are cartesian coordinates. The corresponding (more general) operation for a curved space is to displace the fields from the point $x^{k}$ to the point $x^{k}+c^{k}(x)$ in a coordinate-invariant way. This is achieved by substracting from a field quantity its Lie derivative along the vector field $\xi^{k}(x)$. Now the action

$$
\mathrm{S}=\int \mathrm{d} t ! \int \mathrm{d}^{3} x\left(\pi^{i j} \dot{g}_{i, j}-\mathrm{N}^{\prime \prime} \mathscr{H}_{i 2}\right)+\mathrm{P}^{1}\left[g_{i j}\right]_{1}^{\prime}
$$

is invariant under such a transformation cven if $\xi^{k}$ behaves as $r^{+1}$ at infinity (which is the case for an asymptotic rotation). Note that we have set $\alpha^{\prime \prime}=\delta_{1}^{\prime 2}$ and $\beta^{\prime \prime \prime \prime}=\mathrm{O}$ in $(7.5 \mathrm{I})$ in order to get just a pure Hamiltonian generating changes corresponding to a Minkowskian time displacement at infinity.

Knowing that the action is invariant, the next step is to follow the lines of Noether's theorem and rearrange the variation of the action in the form $\delta S=\int_{i_{1}}^{l} \mathrm{~d} t \underset{\mathrm{d} t}{\mathrm{~d}} \mid \mathrm{d}^{3} x\left(\pi^{i k} \delta g_{i k}\right.$ terms vanishing by the equations of motion, If we then insert

$$
\delta g_{i k}=-\left(\xi_{i \mid k}+\xi_{k \mid i}\right)
$$

into (B.13), we obtain

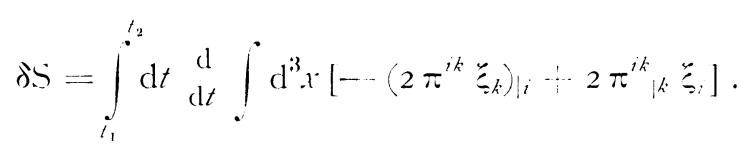

'The second term in (B.It) vanishes clue to constraint $\mathscr{H}_{i}^{\prime} \approx 0$. What is left may be transformed into a surface integral so that $\delta S$ reads

$$
\delta S=\oint \mathrm{d}^{\prime 2} s_{i}\left(-2 \pi^{i k} \dot{\zeta}_{k}\right)_{t_{2}}^{l,}
$$

If we set $\xi_{k} \longrightarrow \infty \varepsilon_{k}$, a constant, we are dealing with an asymptotic translation. We can then write

$$
\delta S=\varepsilon_{k}\left(\mathrm{P}^{k}\left(t_{2}\right)-\mathrm{P}^{k}\left(t_{1}\right)\right)
$$

with

$$
\mathrm{P}^{k}=-2 \oint \mathrm{d}^{2} s_{i} \pi^{i k}
$$

From (B.I6) we identify $\mathrm{P}^{k}$ given by (B.I7) as the total linear momentum of the system. It is a constant of the motion because the action is invariant under the transformation in consideration, so $\delta S$ in (B.I6) is zero. It is $\mathrm{im-}$ portant to realize that only $\varepsilon_{k}=\xi_{k}(\infty)$ provides nontrivial information. 
The invariance of the action with respect to arbitrary $\xi^{k}(x)$ in the interior is immaterial in this context; $\zeta^{k}(x)$ enters in the equations always multiplied by $\mathscr{P}_{k}$ as in (B.I4), which again reflects the close relation between the invariance of the action under arbitrary changes of the spacetime coordinates and the occurrence of constraints in the Hamiltonian theory. The fact that only $\zeta_{k}(\infty)$ matters is the reason that the linear momentum in general relativity is referred to as the conserved quantity associated with "spatial translations at infinity". Similarly one speaks of the energy as being associated with time translations at infinity, and one associates the angular momentum with spatial rotations at infinity. To find the angular momentum $\mathbf{L}$ one just sets $\zeta_{k, r \rightarrow \sim} \rightarrow \varepsilon_{k i, j} \delta \varphi^{i} x^{i}$ in the above reasoning and replaces (B.16) by $\delta S=\delta \varphi^{\prime}\left(\mathrm{L}_{j}\left(t_{2}\right)-\mathrm{L}_{j}\left(t_{1}\right)\right)$. This discussion shows also that the concepts of energy, momentum and angular momentum have no meaning for a closed universe.

4. Relation of Eq. (4.16) to Eq. (1.8.3).

To relate Eqs. (4.16) to Eqs. (1.83) one notices first of all that the function ${ }^{\prime}$, is the generator of reparametrizations $\sigma \rightarrow f(\sigma)$. Thus in the notation of $(1.83)$ we have

$$
\mathscr{H}_{1}=\dot{\psi}_{2}^{\prime} .
$$

On the other hand the generator of deformations along the normal to the string differs from ${ }^{\prime}$ by a factor which is determined up to a conventional sign from the condition:

$$
\text { (B.Ig) }\left\{x^{\prime \prime}(\sigma), \int \mathrm{d} \sigma^{\prime} \lambda\left(\sigma^{\prime}\right) \mathscr{H}_{\perp}\left(\sigma^{\prime}\right)\right\}\left\{x_{\mu}(\sigma), \int \mathrm{d} \sigma^{\prime} \lambda\left(\sigma^{\prime}\right) \mathscr{H}_{\perp}\left(\sigma^{\prime}\right)\right\}=-\lambda^{2}(\sigma),
$$

for an arbitrary function $\lambda(\sigma)$.

This gives

$$
\mathscr{H}_{1}=(2 \mathrm{~N})^{-1}\left(u^{2}\right)^{-\frac{1}{2}} \dot{u}_{1}
$$

where $N$ is the normalization factor appearing in (4.1).

The functions $\mathscr{H}_{\perp}$ and $\mathscr{H}_{1}$ have the following equal- - brackets

$$
\begin{aligned}
& \left\{\mathscr{H}_{1}(\sigma), \mathscr{H}_{1}\left(\sigma^{\prime}\right)\right\}=\left[\left(u^{2}\right)^{-1}(\sigma) \mathscr{H}_{1}(\sigma)+\left(u^{2}\right)^{-1}\left(\sigma^{\prime}\right) \mathscr{F}_{1}\left(\sigma^{\prime}\right)\right]_{\text {i } \sigma}^{2} \delta\left(\sigma-\sigma^{\prime}\right)- \\
& -2 N^{-1}\left[\left(u^{2}\right)^{-3 / 2}(\sigma) \mathscr{H}_{1}(\sigma) \mathscr{H}_{1}(\sigma)+\right. \\
& \left.+\left(u^{2}\right)-32\left(\sigma^{\prime}\right) \mathscr{H}_{1}\left(\sigma^{\prime}\right) \mathscr{H}_{1}\left(\sigma^{\prime}\right)\right] \frac{i}{i \sigma} \delta\left(\sigma-\sigma^{\prime}\right) \text {. } \\
& \left\{\mathscr{H}_{1}(\sigma), \mathscr{H}_{1}\left(\sigma^{\prime}\right)\right\}=\mathscr{H}_{1}(\sigma) \frac{\hat{\sigma}}{\hat{\sigma} \sigma} \delta\left(\sigma-\sigma^{\prime}\right), \\
& \left\{\mathscr{H}_{1}(\sigma), \mathscr{H}_{1}\left(\sigma^{\prime}\right)\right\}=\left[\mathscr{H}_{1}(\sigma)+\mathscr{H}_{1}\left(\sigma^{\prime}\right)\right] \frac{\partial}{\partial \sigma} \delta\left(\sigma-\sigma^{\prime}\right) .
\end{aligned}
$$


Eqs. (B.22) and (B.23) have the same form as $(1.83 b, c)$. This should be the case since these relations just characterize $\mathscr{H}_{1}$ and $\mathscr{H}_{1}$ as being respectively scalar and vector densities in the one-dimensional space of the string. If we notice now that the metric tensor along the string has just one component gi1 given by

$$
g_{11}=\left(g^{11}\right)^{-1}=u^{2}
$$

we see that (B.2 I) agrees with (I.8.3a) but does so only z'eakly. The cliscrepancy comes from the second term in the right hand side of (B.2I):

$$
\begin{gathered}
-2 \mathrm{~N}^{-1}\left[\left(u^{2}(\sigma)\right)^{-3 / 2} \mathscr{H}_{1}(\sigma) \mathscr{H}_{1}(\sigma)+\right. \\
\left.+\left(u^{2}\left(\sigma^{\prime}\right)\right)^{-3 / 2} \cdot \mathscr{H}_{1}\left(\sigma^{\prime}\right) \mathscr{H}_{1}\left(\sigma^{\prime}\right)\right]{ }_{\partial \sigma}^{\partial} \delta\left(\sigma-\sigma^{\prime}\right) .
\end{gathered}
$$

Note however that espression (B.25), being quadratic in the constraints, has weakly ranishing brackets with ererything. Thus the bracket relations (B.2I)-(B.23) still ensure that the dynamical evolution of the string is "path independent" in the sense of Section (I.E). A similar analysis shows that one can replace the factor $\left(u^{2}\right)^{-1}$ in $(\mathrm{B} .2 \mathrm{O})$ by - $\left(\mathscr{P}^{2} / \mathrm{N}^{2}\right)^{-1}$ (obtained by solving $\bigcup_{:}=0$ for $u^{2}$ ), and get an equally $\operatorname{good} \mathscr{H}_{1}$. Such a modification in $\mathscr{H}_{\perp}$ results in fact in the addition to the right hand side of $(\mathrm{B} .2 \mathrm{I})$ of another term quadratic in the constraints. 


\section{REFERENCES}

ABERS E. S. and B. IV. LEE (I973) - "Physics Reports", $9 C_{\text {; }}$ I.

ABRAh.M R. and J. E. MARsinex (I $\left.9^{5} 7\right)$ - Foundations of Mechanics, Benjamin, New York. ANIEROAN J. I. (1958) - " Phys. Rev.", III. 905.

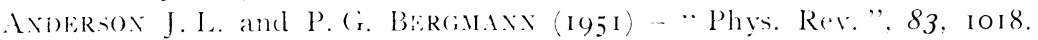

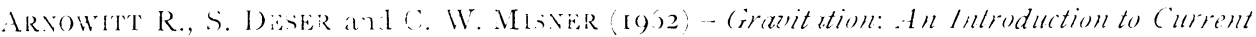
Research |L. Witten. Eal.|. Wiley. New York.

ARNowitt R. and S. I. Fickier (I9)2 - "Phys. Rev.", I27. I821.

BALERLEIN R.F. (I962) -- " Phys. Rev.", I26, 1864.

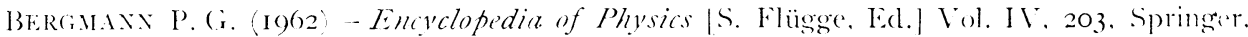
Barlin.

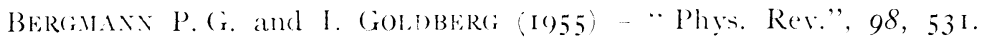

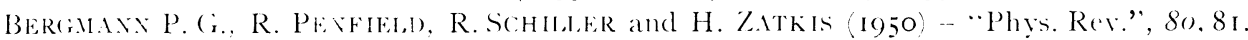

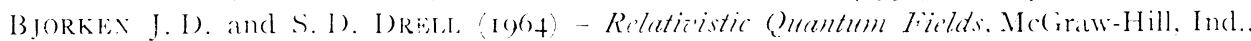
New York.

BROWER R. (. (1972) - "Phys. Rev.", I) 6, I655.

(:HOOLET-BRIHAT (1972) - “( $\therefore$ R. Acad. Sc. Paris". 274, 682.

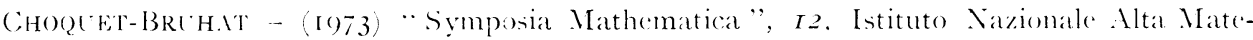
matica, Bologna.

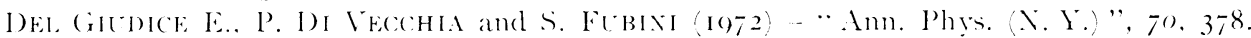
D)EWITT B.S. (I958) unpublished.

D)FWITT B.S. (1959) unpublished.

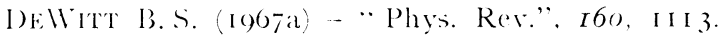

1)EIVITT B.S. $(\mathrm{I}(96)-\mathrm{b})$ - "Phys. Rev." I62, I195.

DIR.AC: P. A.M. (19+8) - "Phỵs. Rev.", 73. I092.

DIRAC P. A. M. (1949a) … Rer. Mod. Phys.", $21,392$.

I)IRAC P.A.M. (I949b) - “ The Dynamical Theory of Fields, Classical and Quantum" Canadian Mathematical Congress, Second Summer Seminar, held at the Eniversity of British Columbia (August. 1949);

I)IRAC P. A. .I. (1950) - - Canad. I. Math.", 2, I 29 ).

DIRAC P.A.M. (I95I) - "Canad. I. Math.", 3, I.

DIR.AC: P. A. M. (1058a) - Proc. Roy. Soc.". 12 126.326.

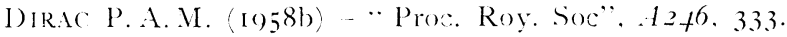

I)IRAC: P.A.M. (I9580) - "Phys. Rer.", II 4. 924.

DIRIC P. A. M. (10)(0) - "Proc. Roy. Soc.". 4257, 52.

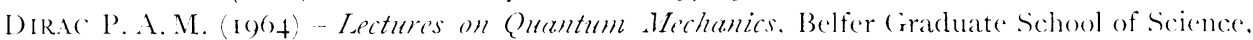
Yeshiva lniversity. Xew York.

I)Irac P. A. M. (1969) - in Contemporary Physics: Trieste Svmposimm a 968 , Vol. I, 539. International Atomic Energy Agency, Vienna.

EISENHART L. P. (1926) - Riemantan (jom tru. Princeton Iniversity, Princeton.

FADDEE L. (I969) - "Teoreticheskaya i Matematicheskaya Fizika". I. 3; FEnglish translation in "Theorsical and Mathematical Physics", I, I (1970)|.

FADIEEV L. and V. N. POPOY (I967a) - " Phys. Lett.", $25 B, 29$.

FAnIEEV L. and V. N. POPOV IG Kiev preprint ITF 67-36 /English translation available as XAI -THY-57 by I). (iordon and B. IV. Lee].

Fubini S., A. J. Haxsox anp R. JACkiw (I973) - "Phys. Rev.", D 7, i 732.

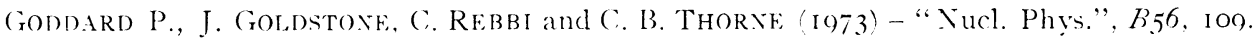
Goddard P., A.J. Haxsox and G. Poxzaxo (1975), - "Nucl. Phys.", B89. 76. 


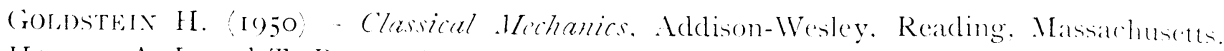

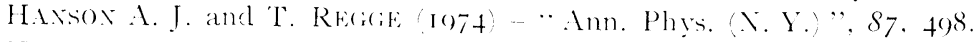

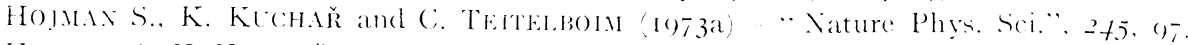

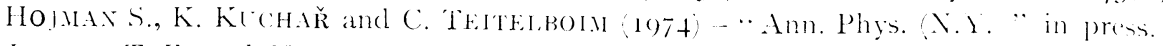

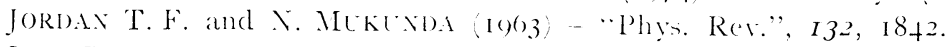

Jost R. $(1964)-\cdots$ Rev. Mod. Phys.", 36, 572.

KoGl'T J. B. and I). E. SOPER (I970) - "Phy's. Rer.", D I, 290 I.

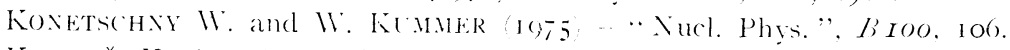

KІCHǍ̆ K. (I97I) - Princeton Iniversity lecture Notes, unpublished.

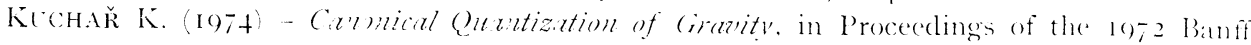
Summer School. Dordrecht. Reidel.

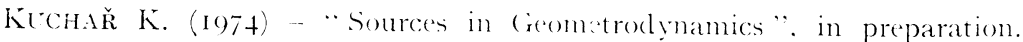

KlMmer W. (IgOI, - "Acta Physica Austriaca". If, If).

KUNDT W. (Ig66) - ".springer Tracts in Modern Physics", fo, ro;.

KLXZLE H. P. (1969) - "Ann. Inst. Henri Poincare". A I I, 393.

LANDAC L. I). and E.M. LAFSHITZ (197) -.. The Classic Theory of Findds. translated by M. Hamermesh, Addison- Wesley, Reading. Massachusetts and Pergamon, I.ondon. I.IChNerowicz A. (I94t) - - J. Math. Pure Appl.", 23, 37.

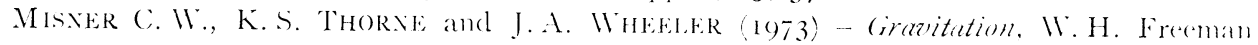
and Co., San Franciseo.

MOHAPATRi R. N. (197la) - "Phys. Rev.", 1) t. 378.

MOHAP.TRA R. N. (107Ib) - "Phys. R(N.", I) t, 2215.

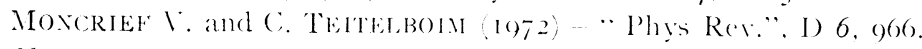

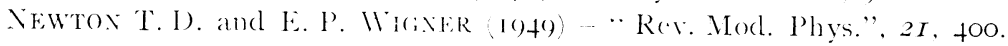

O'Murchadha $X$ and J. II. YORK IO73) “ J. Math. Phys.", If. I 551.

PEIERLS R. E. (I952) - "Proc. ROy. SOC." i2If, I43.

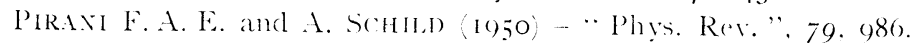

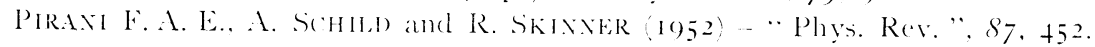

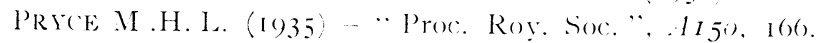

PRYCE M. H. L. 1948 - "Proc. Roy. Soc. ". 1 195, 02.

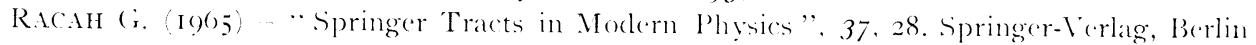
RebBi (C. (1974) - - Physics Reports", I2C: I.

RegGe T. and (. Teiteiboly - "Ann. Phy's. A. Y. " 88, 286.

SCHWINGER J. (I95Ia) - " Phy's. Rev,", 82, gIt.

SCHWINGer J. (195Ib) - " Proc. Natl. Acad. Sci. I. S." "37, 452.

SCHWIAGER J. (1953) - "Phys. Rev.", 9I. 713.

SCHWINGER J. (1962a) -- "Phys. Rev.". I 25. IO+3.

SCHWINGER J. (I062b) - - Phýs. Rev.", I27. 324.

SHANMUgadHASAN S. (1903) - - Proc. Camb. Philos. Soc.", 59. 7t.3.

SHANMUGADHASA S. (I973) - J. Math. Phys. “. If. 677.

SyMANZIK K. (19;I) - " Lectures on Lagrangian Quantum Field Theory ", unpublished DESY Internal Report $\mathrm{T}-7 \mathrm{I}-1$.

Teitelbon C. (1973a) - "The Hamiltonian Structure of Spacetime", l'h. I). Thesis. Princeton Eniversity.

Teitelboin (. (1973b) - "Ann. Phys. ( A. Y.) ", 79, $5+2$.

Touboclas E. (I973) - "Phys. Rev.", I) 8.2736 .

ITIYAMA R. (1956) - "Phys. Rev.", IOI, I597.

WeINBERG S (I973) - "Phys. Rev", I) 7, IO68.

YANG C. N. and R. MILLs (195t) - "Phys. Rev", 96, I9I.

YORk J. W., (I972) - "Phys. Rer. I.tt.", 28, 1082. 